\title{
The Influence of Taiwan Electronic Production Company's Proprietary Data Products on the Interaction of Company Effectiveness
}

\author{
Shih-Yung Wei ${ }^{1}$, Li-Wei Lin ${ }^{2}$ \\ ${ }^{1}$ Business School of Yulin Normal University, Yulin, China \\ ${ }^{2}$ The School of International Business, Zhejiang Yuexiu University, No. 428 Kuaiji Road, Yue Cheng \\ District, Shaoxing 312000, China
}

$\begin{array}{ll}\text { Article Info } & \begin{array}{l}\text { Abstract } \\ \text { Purpose: The purpose of this study is to explore the unique interaction of } \\ \text { Article history: }\end{array} \text { companies in Taiwan from } 1998 \text { to } 2017 \text { and the influence of the degree of } \\ \text { Received:06 June } 2021 & \begin{array}{l}\text { international industry on the effectiveness of the company. } \\ \text { Approach/Methodology/Design: We used time series methods to investigate } \\ \text { and research. This study only has a significant impact on the upstream } \\ \text { Accepted: 14 August } 2021\end{array} \\ \text { industry of the electronics industry, and there is no significant development in } \\ \text { the middle and lower reaches of the industry. This is a very strange situation } \\ \text { in Taiwan. }\end{array}$

\section{Introduction}

Taiwan is one of the most important scientific and technological towns in the world, and its technology industry is more than half of the listed companies, so this study focuses on the listed companies in Taiwan. However, the position of enterprises in the industrial chain is different, and the investment and emphasis on the management factors will also be different. In this study, you focus on the electronic industrial chain to explore the impact of (firm-specific advantages), a proprietary attribute of the company, on the performance of the company. The exclusive advantage of the company is considered to be one of the important factors affecting the operating performance of the enterprise, and the research generally supports that the advantage of the manufacturer has a positive impact on the performance of the manufacturer (Delios and Beamish, 1999 (Morck and Yeung, 1991). Other scholars believe that the factors that help to improve the performance of manufacturers are the advantages of manufacturers (such as R \& D, advertising and capital-intensive) (Jung, 1991). The proprietary assets (Firm-Specific Asset) owned by manufacturers are the most important. The resource (Dess, Gupta, Hennart, and Hitt, 1995). According to the resource base perspective (Resource-Based View, RBV) (Barney, 
1991), the company's unique resources and proprietary assets gain a competitive advantage and generate persistent rewards for excellence (Barney, 1991; Rugman and Verbeke, 2002). These resources may include brand names, skilled labor, scientific and technological knowledge, and efficient production processes (W Ernerfelt, 1984). These resources, such as assets, know-how and skills are difficult to formalize and replicate by competitors, so they can be used for excellent compensation, and these resources are called company-specific assets.

At present, R \& D intensity, capital intensity and marketing intensity are used to measure company-specific assets (Caves, 1971; Jung, 1991; Morck and Yeung, 1991; Hitt et al., 1997; Delios and Beamish, 1999; Kotabe et al., 2002; Zeng et al., 2009). Or just explore the impact of R \& D intensity and marketing intensity on corporate performance (Qian) And Wang, 1999; Lu and Beamish, 2004; Chari et al., 2007; Bae et al., 2008; Ravichandran et al., 2009), but the impact of capital intensity on corporate performance is not taken into account. In addition, compared with the large multinational enterprises in Europe and the United States, the scale of (multinational corporations, MNCs), Taiwan enterprises is relatively small. In addition to internationalization, Taiwan enterprises have special attributes. Can assets improve the performance of manufacturers? This is the research problem that this study wants to clarify very much.

In real variables, Morck and Yeung (1991) use a variety of assets to represent the company's exclusive advantage (FSAs). These assets include technical know-how, marketing capabilities, and customer satisfaction, effective and exclusive management. The specific assets, as defined in Delios and Beamish (1999), refer to the technology and marketing assets owned by a manufacturer, both in terms of research and development (the ratio of $\mathrm{R} \& \mathrm{D}$ expenditure to total sales amount) and advertising-intensive (advertising support) Represented by the ratio of the total pin amount). The capacity of innovation and marketing used in Tt et al. (1997) and Kotabe et al. (2002) is also defined in R \& D intensive and advertising-intensive, and Gruba (1987) uses sales and general management costs (Sailing, General and Administrative Expenses, SG \& A) represents the proportion of total sales to agent marketing or marketing strength. because it is difficult to define. The variables that respond to effective management include the use of different proxy variables to measure management quality. Rugman and Verbeke (2002) integrates the theory of (FSAs), internalization of the company's exclusive advantage and the resource base point of view, and holds that the company's proprietary advantage should be regarded as a collection of knowledge, in the form of assets and learning ability. Even special relationships with external roles. Jung (1991) incorporates the company's capital intensity into the company's exclusive advantage (FSAs), uses R \& D intensity, capital intensity and marketing intensity to measure the company's proprietary assets. Li (1991) 5) use the company's property, plant and equipment as a proportion of total sales to measure capital intensity. Chari et al. (2007) and Ravichandran et al. (2009) proprietary assets are measured in terms of IT investment or IT strength. To sum up, the relevant literature measures the (firm-specific assets) (Bae et al. of the company's proprietary assets in terms of R \& D intensity, marketing intensity, capital intensity and IT intensity. 2008; Lu and Beamish, 2004 1991年)

On R \& D intensity (Research \& Development Intensity, RDI), companies can use product design or research development to improve performance and manufacturing process. If the company has excellent product design, it can obtain a differentiation advantage from competitors and can get a better reward. Similarly, innovation in the process can reduce production costs and improve production quality relative to competitors. As a result, innovation responds to R \& D capabilities that enable companies to achieve operational efficiency (Hitt et al., 1994). When companies enter international markets, operational innovation becomes important, and

Copyright (C) 2020, Journal of Advanced Research in Economics and Administrative Sciences (JAREAS), Under a 
companies can use their innovative products to generate additional revenue, or further reduce production costs, use their manufacturing processes and achieve economies of scale to profit.The past studies have used $\mathrm{R} \& \mathrm{D}$ strength to measure innovation and believe that innovation or process innovation (Kotoabe et al., 2002), whether it is a product, can improve the operating efficiency of the manufacturer (Supt et al.,1994), so, The study also found that the relationship between $\mathrm{R} \& \mathrm{D}$ strength and the manufacturer's performance has a positive regulation of internationalization and the company's performance (Delios and Beamish,1999; Kotabe et al.,2002; Lu and Beamish,2004; Ba et al., 2008). Hirschey and Connolly (2005) found that research and development expenditure of US vendors had a positive impact on their market value(measured by TobinsQ); Compared with small companies, the R\&D investment of large companies has great influence on TobinsQ.

While international corporate literature generally supports the positive relationship between R \& $\mathrm{D}$ and vendor performance, some scholars have found a reverse relationship between $\mathrm{R} \& \mathrm{D}$ activities and corporate performance (Chan, Martin, and Kenser, 1990; Mank and Nystrom, 2001). The investment return to challenge R \& D activities may thus be rather low (Rouse and Boff, 1998). Franzen and Radhiakrishnan (2009) Using the COMPUSTAT database 1 From 982 to 2002, a total of 47167 annual data were collected to explore the influence of research development expenditure on the stock value of profitable companies (profit firms) and lossmaking companies (loss firms). The empirical results show that the investment of research and development has a negative correlation with the stock price of profitable companies, that is to say, the investment of research and development has a negative impact on the value of profitable companies.

In addition to the $\mathrm{R} \& \mathrm{D}$ and innovation ability of the manufacturer, the unique marketing ability, such as brand management or trademark, is one of the important advantages of the manufacturer in the "proprietary asset" (proprietary assets) referred to in Caves (1996). As the market becomes more globalized across national boundaries, manufacturers with more marketing capabilities can produce better performance than those with less investment (Caves, 1996 / Hennart, 1991; Lall, 1980). Therefore, manufacturers expect higher advertising and marketing costs to be able to overseas markets generate higher sales (Kotabe et al., 2002).

Delios and Beamish (1999) it is found that advertising expenditure has a negative relationship with subsidiary performance. Riram and Sapienza (1991) discuss the impact of marketing ability on the performance of small and medium-sized exporters, but does not find evidence that marketing activities contribute to performance. Qian (2002) puts marketing intensity into the model as a control variable to explore, and also obtains that it has no significant impact on manufacturer performance, so on the whole, Empirical research on how advertising investment affects the performance of international enterprises has not yet been highly consistent.

In terms of capital intensity, by investing in plant and equipment expenditure, the company expands production capacity to achieve economies of scale, reduce unit production costs, and gain a competitive advantage in cost to obtain more orders. And products with lower production costs can be sold to other countries with higher production costs for remuneration. Therefore, it is considered that if the company has a higher capital intensity, it will achieve better performance from the internationalization strategy.

There is very little interaction between the "specific assets" and the effect of the company's performance, and the interaction between the variables will be more or less, especially if the

Copyright (C) 2020, Journal of Advanced Research in Economics and Administrative Sciences (JAREAS), Under a 
"should" will exist in the resource allocation of the" specific assets", Therefore, this study is to study the situation and compare the change of interaction under the change of time.

\section{Methodology and Procedures}

\section{Research Data}

In this study, the Asian financial turmoil was used as the cutting point to study the interaction between the specific assets of Taiwan electronics chain companies and the performance of Taiwan electronics chain companies in the past 20 years from 1998 to 2017. In the course of the study, the abnormal data are deleted, so the data in this study are unbalanced. The industrial structure of Taiwan's electronic industrial chain from 1998 to 2017 is shown in Table 1. It can be found that the number of samples in this study is 15098 / year, of which 2624 are upstream (semiconductor), 5936 / year (electronic parts, optoelectronic), downstream (computer and surrounding, electronic access, others). There are 6538 electronic, communication networks, information services). Therefore, this study not only discusses the impact of company-specific assets on corporate performance interaction, but also further discusses the impact of export ratio on corporate performance, that is, the impact of internationalization on corporate performance and the impact of other control variables on corporate performance.

Table 1: List of electronic industry chain structure of Listed Companies in Taiwan from 1998 to 2017

\begin{tabular}{|c|c|c|c|c|c|c|c|c|c|c|c|c|c|}
\hline year & 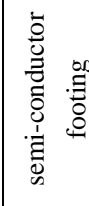 & Add up & 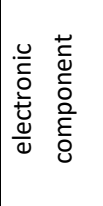 & 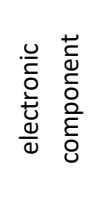 & Add up & 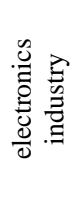 & 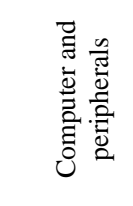 & 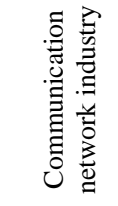 & 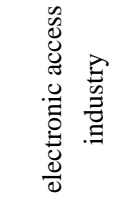 & 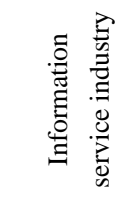 & 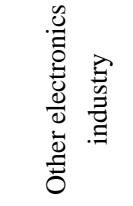 & $\begin{array}{l}\text { Add } \\
\text { up }\end{array}$ & total \\
\hline 1998 & 49 & 49 & 68 & 35 & 103 & & 52 & 25 & 13 & 10 & 58 & 158 & 310 \\
\hline 1999 & 67 & 67 & 89 & 47 & 136 & 1 & 61 & 40 & 17 & 13 & 78 & 210 & 413 \\
\hline 2000 & 76 & 76 & 116 & 60 & 176 & 1 & 72 & 51 & 22 & 24 & 97 & 267 & 519 \\
\hline 2001 & 97 & 97 & 131 & 72 & 203 & 2 & 76 & 58 & 30 & 27 & 117 & 310 & 610 \\
\hline 2002 & 109 & 109 & 153 & 82 & 235 & 3 & 89 & 65 & 38 & 31 & 137 & 363 & 707 \\
\hline 2003 & 117 & 117 & 166 & 87 & 253 & 3 & 94 & 70 & 38 & 31 & 150 & 386 & 756 \\
\hline 2004 & 120 & 120 & 170 & 93 & 263 & 3 & 96 & 71 & 40 & 30 & 131 & 371 & 754 \\
\hline 2005 & 119 & 119 & 177 & 94 & 271 & 2 & 93 & 72 & 42 & 29 & 104 & 342 & 732 \\
\hline 2006 & 127 & 127 & 185 & 101 & 286 & 2 & 101 & 74 & 43 & 31 & 81 & 332 & 745 \\
\hline 2007 & 142 & 142 & 192 & 119 & 311 & 2 & 101 & 76 & 45 & 30 & 73 & 327 & 780 \\
\hline 2008 & 142 & 142 & 193 & 130 & 323 & 2 & 105 & 77 & 43 & 30 & 75 & 332 & 797 \\
\hline 2009 & 151 & 151 & 198 & 139 & 337 & 2 & 108 & 82 & 43 & 27 & 78 & 340 & 828 \\
\hline 2010 & 158 & 158 & 206 & 157 & 363 & 2 & 107 & 83 & 42 & 30 & 81 & 345 & 866 \\
\hline 2011 & 160 & 160 & 209 & 174 & 383 & 2 & 109 & 84 & 40 & 30 & 80 & 345 & 888 \\
\hline 2012 & 163 & 163 & 213 & 172 & 385 & 3 & 104 & 84 & 40 & 29 & 81 & 341 & 889 \\
\hline 2013 & 163 & 163 & 220 & 169 & 389 & 3 & 108 & 87 & 41 & 28 & 80 & 347 & 899 \\
\hline 2014 & 168 & 168 & 222 & 172 & 394 & 3 & 112 & 91 & 40 & 32 & 78 & 356 & 918 \\
\hline 2015 & 172 & 172 & 216 & 166 & 382 & 3 & 110 & 93 & 39 & 31 & 76 & 352 & 906 \\
\hline 2016 & 161 & 161 & 217 & 155 & 372 & 3 & 109 & 93 & 40 & 36 & 76 & 357 & 890 \\
\hline 2017 & 163 & 163 & 219 & 152 & 371 & 3 & 108 & 93 & 37 & 38 & 78 & 357 & 891 \\
\hline 总计 & 2624 & 2624 & 3560 & 2376 & 5936 & 45 & 1915 & 1469 & 733 & 567 & 1809 & 6538 & 15098 \\
\hline
\end{tabular}

Copyright (C) 2020, Journal of Advanced Research in Economics and Administrative Sciences (JAREAS), Under a 


\section{Research Variable}

\section{Independent Variable- Corporate Proprietary Assets}

When firms obtain differential benefits from international expansion, they should maximize the benefits of internationalization and minimize the costs of internationalization with their capabilities. Such capabilities could be considered proprietary corporate assets. Proprietary corporate assets include a firm's R\&D and its capital strength (Jung, 1991). Therefore, this study believed that a firm's R\&D and capital strength could enable the firm to obtain benefits from an internationalization strategy. Kotabe et al. (2002) were of the view that a firm with R\&D and marketing capabilities could be able to obtain higher prices for their innovative products or expand overseas markets to reach economies of scale with its $R \& D$ and marketing capabilities to reduce production costs. Based on the above discussion, this study proposed the following hypothesis: proprietary corporate assets (including R\&D expense ratio, marketing expense ratio, and fixed asset growth rate) could reduce the costs of products, improve the added value of products, and improve firm performance through internationalization activities.

\section{R\&D Expense Ratio}

R\&D intensity is used to measure innovations, and both product innovations and process innovations are considered helpful to improve the operation efficiency of firms. Research has also found that R\&D intensity is positively related to firm performance (Bradley, Jarrell \& Kim, 1984; Morck et al., 1988; Titman \& Wessels, 1988; Crutchley \& Hansen, 1989; McConnell \& Servaes, 1990; Hermalin \& Weisbach, 1991; Jensen et al., 1992; Hirschey \& Weygandt, 1993; Klette, 1996; Delios \& Beamish, 1999; Kotabe et al., 2002; Lu \& Beamish, 2004; Bae et al., 2008; Missaka, 2015; Josheski \& Sopova, 2013; Shih-Yung et. al., 2017).

However, some scholars have found that $R \& D$ activities are negatively related to firm performance (Chan, Martin, \& Kensinger, 1990; Rouse \& Boff, 1998; Mank \& Nystrom, 2001; Franzen \& Radhakrishnan, 2009), and thus stated that the investment incomes of R\&D activities may be quite low, that is, R\&D investment has negative effects on the value of profitable firms. Scherer (1965) and Lev and Aboody (2001) demonstrated that the benefits of R\&D activities have the time lag effect. This research comprehensively discusses the previous research results obtained by scholars and explores the long-term effects of R\&D intensity on firm performance. The $R \& D$ expense ratio is used as the proxy variable of $R \& D$ intensity.

$$
\mathrm{R} \& \mathrm{D} \text { Expense Ratio }(\mathrm{RD})=\frac{\mathrm{R} \& \mathrm{D} \text { expense }}{\text { Sale revenues }}
$$

\section{Growth Rate of Fixed Assets (LA)}

Agrawal and Knoeber (1996), Titman and Wessels (1988), and Shih-Yung Wei et. al. (2017) noted that the higher the growth rate of fixed assets is, the more opportunities for future investment and growth a company has. The growth rate of fixed assets is also an indicator of corporate performance.

$$
\mathrm{LA}=\frac{\text { (Total fixed assets of the year }- \text { Total fixed assets of the previous year) }}{\text { Total fixed assets of the previous year }}
$$

Copyright (C) 2020, Journal of Advanced Research in Economics and Administrative Sciences (JAREAS), Under a Creative Commons Attribution 4.0 International License 


\section{Strength in Marketing}

Morck and Yeung (1991) found that under international M\&As of major firms, investors have a positive impact on the market value of firms. Kravis and Lipsey (1992) demonstrated that both firms' R\&D investment and advertisement costs could contribute to improving export performance. Kotabe et al. (2002) and Lu and Beamish (2004) demonstrated that higher advertisement and marketing costs could contribute to improving the sales of international firms in overseas markets. Shah, Stark, and Akbar (2009) utilized cross-sectional data from 1990 to 1998 in the UK and 9,752 observed values to research the value relevance of the main media advertisement costs of ACNielsen MEA, and the results showed a positive correlation between advertisement costs and the market value of the firm. The marketing expense ratio refers to the marketing cost of a firm (including costs for publishing advertisements in newspapers and magazines, on television stations, at cinemas and theaters, advertisement designs and productions from advertising agencies, touring propaganda, color advertising boards, electric advertising, and printing of advertising materials, as well as free samples and items) within a certain period (generally one year) divided by operating revenue. This ratio is generally expressed as a percentage:

$$
\text { Strength in marketing }(\mathrm{MI})=\frac{\text { Marketing cost }}{\text { Sale revenues }}
$$

\section{Dependent variable -Corporate Performance (Tobin's Q )}

This study adopts Tobin's Q, the most commonly used indicator to measure a company's market performance. La Porta et al. (2002) employed Tobin's Q, but failed to figure out Tobin's Q, because they could not obtain the replacement cost of company assets. As a result, they replaced Tobin's Q with Proxy Q, and the latter was adopted by Claessens et al. (2000). Proxy Q is measured as follows:

$$
\begin{aligned}
& \text { Tobin's } Q=\frac{\text { Market value of equity }+ \text { market value of debts }}{\text { Asset replacement cost }} \\
& \text { Proxy } Q=\frac{\text { Market value of equity (commonstocks + special stocks) }+ \text { book value of debts }}{\text { Book value of assets }}
\end{aligned}
$$

\section{Control Variables}

\section{Degree of internationalization (FS)}

By measuring the degree of internationalization with the ratio of foreign sales to total sales (FSTS), Kafouros et al. (2008); Hsu and Pereira (2008); Bae et al. (2008); Gaur and Kumar (2009); Filatotchev and Piesse (2009), and Brouthers et al. (2009) believed that a higher degree of internationalization has more positive effects on firm performance.

Degree of internationalization= ratio of foreign sales to total sales

\section{Scale of Company (SC)}

Firms with a large scale can generally be regarded as having the capability to acquire a profit margin above the normal level, as compared with general firms. Therefore, such firms are able to operate in an imperfect market and acquire a higher excess profit by leveraging their monopoly or oligopoly strength. Furthermore, firms with a large scale may have access to funds with a low cost in the capital market or operate in the market with a low cost due to risk diversification.

Copyright (C) 2020, Journal of Advanced Research in Economics and Administrative Sciences (JAREAS), Under a 
In respect of the impact of the scale of a company on performance, it is easier for firms with a larger scale to utilize the advantage of economies of scale to result in good operating performance. Therefore, scale of company was defined as a control variable. Measurement of the scale of company includes total assets, total operating revenue, and number of employees (Kotabe et al., 2002; Lu \& Beamish, 2004; Chari et al., 2007; Bae et al., 2008; Ravichandran et al., 2009). Generally, the total assets or operating cost of a firm at natural logarithms is defined as a proxy variable. Therefore, in this study, the carrying amounts of the total assets of the sample firms at natural logarithms were used as proxy variables.

$$
\text { Scale of company }=\log (\text { total assets })
$$

\section{Debt-Asset Ratio (D/A; DA)}

Myers (1977), Jensen (1986), Morck, Shleifer, and Vishny (1988), Stulz (1990), Shih-Yung Wei et al. (2017) argued that the debt-asset ratio, on the one hand, implies the information of corporate tax shields; on the other hand, according to the Pecking Order Theory, the higher the debt-asset ratio is, the lower the rate on investment is, and the smaller the corporate value will be.

$$
\text { Debt }- \text { asset ratio }=\frac{\text { Book value of debt }}{\text { Book value of asset }}
$$

\section{Firm Age; AG}

The firm age of this study refers to the natural firm age, and so its calculation mode is as follows:

Firm age $=\frac{(\text { Date of data collectior }(\text { supposed December31 of that year })-\text { Date of establishment })}{365}$

\section{Board Structure}

This variable is presented in three forms in this study: board size (BSIZE; BS), ratio of external directors (PE), and concurrent positions of directors (CP). Yermack (1996) and Shih-Yung et al. (2017) studied the relationship between board size and corporate performance. The empirical results of their studies show that board size and performance are negatively correlated - that is, a smaller board of directors can better supervise managers to raise the corporate value. Fich and Shivdasani (2005) found when most of the members of the board of directors are concurrent directors of three or more other companies that corporate performance will be undermined; Core, Holthausen, and Larcker (1999) and Shivdasani and Yermack (1999) proposed when members of the board of directors hold multiple positions concurrently that they cannot effectively supervise the managers. However, the empirical results of some foreign literature hold the opposite view. For example, Ferris, Jagannathan, and Pritchard (2003) found no evidence to indicate when most directors hold three or more positions that they will evade their responsibilities - that is, evading the responsibility of supervising managers. Yermack (2004) noted when most board directors hold three or more positions that they will still fulfill the responsibility of supervising managers.

Regarding a board of directors composed by external and insider directors, from the supervisory point of view, although external directors have less information to supervise managers, they can play a more independent supervisory role, because of their independent status. Internal directors who hold positions within the company will have more information to supervise managers, but

Copyright (C) 2020, Journal of Advanced Research in Economics and Administrative Sciences (JAREAS), Under a 
their potential conflicts of interest with managers may subject them to the control of managers or make them more inclined to collaborate with managers to adopt strategies that compromise corporate interests. Fama (1980) and Baysinger and Hoskisson (1990) believed that external supervisors, who are independent inside the company and boast know-how, are hired by companies in the hope that they use their professional knowledge to improve corporate performance. Therefore, the higher the ratio of external directors in a company is, the more effective the supervision can be, and the better the corporate performance can be achieved through their professional knowledge.

$$
\begin{aligned}
& \text { Scale of board of directors }=\text { Total seats of directors } \\
& \text { Ratio of external directors }=\frac{\text { Seats of external directors }}{\text { Total seats of directors }}
\end{aligned}
$$

The concurrent position of directors is a virtual variable. If more than half of the board of directors assume three or more positions (including their position in the sampled company), then the variable is 1 and otherwise 0 .

Due to problematic data acquisition, the definition of positions is mainly those directors and managers present in the annual reports of listed companies.

\section{Proportion of Pledged Shares by Directors (Pledge; PL)}

This proportion is one of the commonly used indicators for corporate governance. Yeh and Lee (2001) and Shih-Yung Wei et al. (2017) argued that the higher the proportion is of pledged shares by major shareholders, the deeper their involvement in the stock market is, and the worse the corporate performance will be.

$$
\text { Proportionof pledge shares by directors }=\frac{\text { Quantity of pledge by directors }}{\text { Total shares by all directors }}
$$

The estimated impact of the control variables in this study on corporate performance is shown in Table 2.

\section{Research Method}

This study explores the impact of Long-term Effects of Research and Development on corporate

\begin{tabular}{|c|c|c|c|}
\hline Variable & Definitions & expected & notes \\
\hline \multicolumn{4}{|l|}{ Dependent variable } \\
\hline \multicolumn{4}{|c|}{ Market value of equity (commonstocks + special stocks) + book value of debts } \\
\hline Tobin's Q & Book value of assets & & \\
\hline \multicolumn{4}{|l|}{ Independent variable } \\
\hline $\begin{array}{l}\text { R\&D Expense Ratio } \\
\text { (RD) }\end{array}$ & $R \& D$ Expense Ratio $(R D)=\frac{R \& D \text { expense }}{\text { Sale revenues }}$ & ? & $\begin{array}{l}\text { Morck et al. (1988), Shih } \\
\text { Yung Wei et. al.(2017)... } \\
\text { Morck and Yeung (1991) }\end{array}$ \\
\hline $\begin{array}{l}\text { Strength in marketing } \\
\text { (MI) }\end{array}$ & Strength in marketing $(\mathrm{MI})=\frac{\text { Marketing cost }}{\text { Sale revenues }}$ & + & $\begin{array}{l}\text {,Kravis and Lipsey } \\
(1992), \text { Kotabe et al } \\
(2002) \text {,Lu and Beamish } \\
\text { (2004) and Shah, Stark, } \\
\text { and Akbar (2009) }\end{array}$ \\
\hline
\end{tabular}
performance from 1998 to 2017, and so Panel Data represents the data of this study.

Table2.Summary of definitions of variables and expected effect

Copyright (C) 2020, Journal of Advanced Research in Economics and Administrative Sciences (JAREAS), Under a Creative Commons Attribution 4.0 International License 


\begin{tabular}{|c|c|c|c|}
\hline Variable & Definitions & expected & notes \\
\hline $\begin{array}{l}\text { Growth Rate of Fixed } \\
\text { Assets (LA) }\end{array}$ & (Total fixed assets of the year -Total fixed assets of last year)/Total fixed & lassets of lo & $\begin{array}{l}\text { yegrawal, } \\
\text { (1996) }\end{array}$ \\
\hline \multicolumn{4}{|l|}{ Control variables } \\
\hline Degree & & & $\begin{array}{l}\text { Bae et al. (2008); Gaur } \\
\text { and } \quad \text { Kumar(2009); }\end{array}$ \\
\hline $\begin{array}{l}\text { Internationalization } \\
\text { (FS) }\end{array}$ & $\begin{array}{l}\text { Foreign Sales as a percentage of Total Sales, } \\
\text { FSTS(FSTS) }\end{array}$ & + & $\begin{array}{l}\text { Filatotchev and } \\
\text { Piesse(2009); Brouthers } \\
\text { et al. (2009)、.. } \\
\text { Yeh and Lee (2001) }\end{array}$ \\
\hline Pledge Ratio（PL） & pledge/shares held & - & $\begin{array}{l}\text { Shih Yung Wei et. } \\
\text { al.(2017) }\end{array}$ \\
\hline & & & McConnell and \\
\hline Liability Ratio（DA） & book value of debts/book value of assets & $?$ & $\begin{array}{l}\text { Servaes(1995), Shih } \\
\text { Yung Wei et. al.(2017) }\end{array}$ \\
\hline Scale (SC) & $\ln ($ total assets $)$ & + & $\begin{array}{l}\text { Shih Yung Wei et. } \\
\text { al.(2017) }\end{array}$ \\
\hline Board Size (BS) & seats of directors & - & $\begin{array}{l}\text { Yermack (1996), Shih } \\
\text { Yung Wei et. al.(2017) }\end{array}$ \\
\hline $\begin{array}{ll}\text { Concurrent } & \text { Post } \\
\text { Holding (CP) } & \\
\text { (dummy variable) } & \end{array}$ & $\left\{\begin{array}{lc}1, & \text { half of the directors hold three or more positions } \\
0, & \text { else }\end{array}\right.$ & $?,-$ & $\begin{array}{l}\text { Fich, Shivdasani } \\
\text { (2005), Shih Yung Wei } \\
\text { et. al.(2017) } \\
\text { Fama }\end{array}$ \\
\hline $\begin{array}{l}\text { Proportion of External } \\
\text { Directors (PD) }\end{array}$ & number of external directors / total number of director & & $\begin{array}{l}\text { (1980)与Baysinger and } \\
\text { Hoskisson (1990), Shih } \\
\text { Yung Wei et. al.(2017) }\end{array}$ \\
\hline
\end{tabular}

Panel Data is a form of data that take cross-section data and time sequence into account simultaneously. As a result, if the data for analysis are heterogeneous, then the traditional analysis method of the least square method (OLS) will cause invalid results in the analysis of Panel Data, in that OLS can only process either cross-section or time sequence data. When cross-section and time sequence both exist in the data, OLS ignores the differences between these two, resulting in inefficient estimation results. However, the Panel Datamodel can process data featuring a mixture of heterogeneity and time sequence and can produce more effective estimation results.

The Panel Data model is unable to process all kinds of data featuring a mixture of heterogeneity and time sequence. Whether this model can be adopted should be decided by comparing the general regression model with the mixed regression equation model. The Panel Data model can be basically divided into the fixed effect model and random effect model, both of which have their respective characteristics and applicability. The model type can be selected through a simple judgment. Intuitively, cross-sectional units that are selected without sampling should adopt the fixed effect model; conversely, cross-sectional units that are selected after sampling should adopt the random effect model. However, there is no scientific basis for such judgment. Mundlak (1978) believed that errors will occur if the intercept term of the random effect model correlates with the independent variable. In this case the fixed effect model should be adopted; if the intercept term is independent of the independent variable, then the random effect model should be adopted. To decide on which model, the Hausman Test of Hausman (1978) can be used.

\section{Results and Discussion}

\section{Uni-variable Analysis}

Copyright (C) 2020, Journal of Advanced Research in Economics and Administrative Sciences (JAREAS), Under a 
This unit carries on the narrative statistics analysis, this research sample number is 15098 / year, the upstream (semiconductor) has 2624 / year, the middle reaches (electronic parts, optoelectronics) has 5936 / year, the downstream (computer and its periphery, electronic path, other electronics, communication network, information service) has 6538 / year, the narrative statistics of the research variables can be observed by Table 3、 Table4 and Table 5.

It can be found from Table 3 / 5 that Tobin's Q, R \& D intensity (RD), marketing intensity (MI), capital intensity (LA), board size (BS), board of directors part-time situation (BP), board of directors stock pledge ratio (PL), company size (SC), debt ratio (DA) and company age showing a high narrow peak (leptokurtosis), Others (the degree of internationalization (FS) and the proportion of independent directors (BO) are low broad peak (platykurtic).

Table3. Upstream Industry Description of Statistics

\begin{tabular}{lllllllllllll} 
& $\begin{array}{l}\text { Tobin's } \\
\text { Q }\end{array}$ & RD & MI & LA & BS & BO & BP & PL & SC & DA & AG & FS \\
\hline Obs. & 2624 & 2624 & 2624 & 2624 & 2624 & 2624 & 2624 & 2624 & 2624 & 2624 & 2624 & 2624 \\
\hline Mean & 1.53 & 0.12 & 0.00 & 0.17 & 9.23 & 0.20 & 0.15 & 0.05 & 15.00 & 0.32 & 15.16 & 0.56 \\
\hline Med. & 1.09 & 0.08 & 0.00 & 0.00 & 9.00 & 0.20 & 0.00 & 0.00 & 14.78 & 0.29 & 14.20 & 0.62 \\
\hline Max. & 14.30 & 5.97 & 0.08 & 4.96 & 19.00 & 0.80 & 1.00 & 1.00 & 21.41 & 0.99 & 50.24 & 1.00 \\
\hline Min. & 0.00 & 0.00 & 0.00 & -0.89 & 2.00 & 0.00 & 0.00 & 0.00 & 10.70 & 0.01 & 0.25 & 0.00 \\
\hline Std. & 1.34 & 0.22 & 0.00 & 0.58 & 1.99 & 0.16 & 0.35 & 0.13 & 1.64 & 0.18 & 7.99 & 0.29 \\
\hline Sk. & 3.77 & 12.79 & 9.06 & 3.94 & 0.56 & 0.19 & 2.01 & 3.45 & 0.71 & 0.74 & 0.86 & -0.41 \\
\hline K. & 23.77 & 257.58 & 117.36 & 24.35 & 4.08 & 2.31 & 5.06 & 16.92 & 3.44 & 3.24 & 3.94 & 2.01 \\
\hline
\end{tabular}

Table4. Midstream Industry Description of Statistics

\begin{tabular}{lllllllllllll} 
& $\begin{array}{l}\text { Tobin's } \\
\text { Q }\end{array}$ & RD & MI & LA & BS & BO & BP & PL & SC & DA & AG & FS \\
\hline Obs. & 5936 & 5936 & 5936 & 5936 & 5936 & 5936 & 5936 & 5936 & 5936 & 5936 & 5936 & 5936 \\
\hline Mean & 1.07 & 0.04 & 0.00 & 0.14 & 9.14 & 0.18 & 0.09 & 0.05 & 14.92 & 0.42 & 19.80 & 0.69 \\
\hline Med. & 0.87 & 0.02 & 0.00 & 0.01 & 9.00 & 0.20 & 0.00 & 0.00 & 14.75 & 0.42 & 18.71 & 0.78 \\
\hline Max. & 14.51 & 3.55 & 0.10 & 4.43 & 19.00 & 0.75 & 1.00 & 1.00 & 20.38 & 0.99 & 52.65 & 1.00 \\
\hline Min. & 0.00 & 0.00 & 0.00 & -0.89 & 3.00 & 0.00 & 0.00 & 0.00 & 10.08 & 0.01 & 0.01 & 0.00 \\
\hline Std. & 0.79 & 0.09 & 0.00 & 0.47 & 1.76 & 0.15 & 0.29 & 0.14 & 1.29 & 0.16 & 9.96 & 0.28 \\
\hline Sk. & 5.39 & 20.55 & 9.98 & 3.72 & 0.16 & 0.07 & 2.77 & 3.34 & 0.77 & 0.17 & 0.45 & -1.01 \\
\hline K. & 55.50 & 630.45 & 152.43 & 23.28 & 3.87 & 1.94 & 8.65 & 15.32 & 3.98 & 2.93 & 2.58 & 3.01 \\
\hline
\end{tabular}

Table5. Downstream Industry Description of Statistics

\begin{tabular}{lllllllllllll} 
& $\begin{array}{l}\text { Tobin's } \\
\mathbf{Q}\end{array}$ & RD & MI & LA & BS & BO & BP & PL & SC & DA & AG & FS \\
\hline Obs. & 6538 & 6538 & 6538 & 6538 & 6538 & 6538 & 6538 & 6538 & 6538 & 6538 & 6538 & 6538 \\
\hline Mean & 1.12 & 0.06 & 0.00 & 0.15 & 8.87 & 0.16 & 0.11 & 0.06 & 14.74 & 0.43 & 19.11 & 0.60 \\
\hline Med. & 0.86 & 0.03 & 0.00 & 0.01 & 9.00 & 0.20 & 0.00 & 0.00 & 14.53 & 0.43 & 18.19 & 0.75 \\
\hline Max. & 19.87 & 6.11 & 0.10 & 4.91 & 32.00 & 0.67 & 1.00 & 0.99 & 21.95 & 0.99 & 63.98 & 1.00 \\
\hline Min. & $\mathbf{0 . 0 0}$ & $\mathbf{0 . 0 0}$ & $\mathbf{0 . 0 0}$ & $\mathbf{- 0 . 8 9}$ & $\mathbf{2 . 0 0}$ & $\mathbf{0 . 0 0}$ & $\mathbf{0 . 0 0}$ & $\mathbf{0 . 0 0}$ & $\mathbf{9 . 6 7}$ & $\mathbf{0 . 0 1}$ & $\mathbf{0 . 1 4}$ & $\mathbf{0 . 0 0}$ \\
\hline Std. & $\mathbf{0 . 8 6}$ & $\mathbf{0 . 1 6}$ & $\mathbf{0 . 0 1}$ & $\mathbf{0 . 5 5}$ & $\mathbf{1 . 9 4}$ & $\mathbf{0 . 1 6}$ & $\mathbf{0 . 3 2}$ & $\mathbf{0 . 1 4}$ & $\mathbf{1 . 4 8}$ & $\mathbf{0 . 1 8}$ & $\mathbf{9 . 5 0}$ & $\mathbf{0 . 3 7}$ \\
\hline Sk. & $\mathbf{5 . 6 7}$ & $\mathbf{2 1 . 8 2}$ & $\mathbf{5 . 4 6}$ & $\mathbf{4 . 1 4}$ & $\mathbf{1 . 0 9}$ & $\mathbf{0 . 3 0}$ & $\mathbf{2 . 4 4}$ & $\mathbf{3 . 1 3}$ & $\mathbf{1 . 0 6}$ & $\mathbf{0 . 0 7}$ & $\mathbf{0 . 5 2}$ & $\mathbf{- 0 . 5 4}$ \\
\hline K. & $\mathbf{7 4 . 9 7}$ & $\mathbf{6 3 8 . 3 6}$ & $\mathbf{4 2 . 8 7}$ & $\mathbf{2 5 . 9 1}$ & $\mathbf{9 . 5 3}$ & $\mathbf{1 . 8 8}$ & $\mathbf{6 . 9 7}$ & $\mathbf{1 3 . 9 3}$ & $\mathbf{4 . 7 8}$ & $\mathbf{2 . 4 5}$ & $\mathbf{3 . 1 2}$ & $\mathbf{1 . 6 9}$ \\
\hline
\end{tabular}


In terms of bias, the internationalization degree is left-sided, and the rest are all right-sided. The average and median of central trends do not change substantially.

\section{Bi-variable Analysis}

From the matrix of correlation coefficients in Table 6 、 Table 7 and Table 8, it can be found that most of the correlation coefficients among the explanatory variables are low, and the interaction between them is not significant. Regression analysis will not produce different results from the actual situation, while Scale, Age,D/A ratio and Pledge ratio is negatively correlated with the dependent variable Tobin's $Q$ in the independent variables, and most of these variables are also negatively correlated with other variables. Other variables are positively correlated with Tobin's Q, but the impact of variables and Tobin's Q still needs to be improved. Step by step econometric analysis can be obtained.

Table6. Upstream Industry Correlation Coefficient Matrix

\begin{tabular}{|c|c|c|c|c|c|c|c|c|c|c|c|c|}
\hline & $\begin{array}{l}\text { Tobin's } \\
\text { Q }\end{array}$ & RD & MI & LA & BS & BO & BP & PL & SC & DA & $\mathbf{A G}$ & FS \\
\hline $\begin{array}{l}\text { Tobin's } \\
\text { Q }\end{array}$ & 1.00 & & & & & & & & & & & \\
\hline & ----- & & & & & & & & & & & \\
\hline RD & $\begin{array}{l}0.09 \\
(4.37) \\
* * *\end{array}$ & $\begin{array}{l}1.00 \\
---- \\
----\end{array}$ & & & & & & & & & & \\
\hline MI & $\begin{array}{l}0.07 \\
(3.74) \\
* * *\end{array}$ & $\begin{array}{l}0.20 \\
(10.41) \\
* * *\end{array}$ & $\begin{array}{l}1.00 \\
---- \\
----\end{array}$ & & & & & & & & & \\
\hline LA & $\begin{array}{l}0.10 \\
(5.20) \\
* * *\end{array}$ & $\begin{array}{l}0.01 \\
(0.62)\end{array}$ & $\begin{array}{l}0.06 \\
(3.30) \\
* * *\end{array}$ & $\begin{array}{l}1.00 \\
---- \\
----\end{array}$ & & & & & & & & \\
\hline BS & $\begin{array}{l}0.02 \\
(1.06)\end{array}$ & $\begin{array}{l}-0.09 \\
(-4.78) \\
* * *\end{array}$ & $\begin{array}{l}0.00 \\
(-0.19)\end{array}$ & $\begin{array}{l}-0.05 \\
(-2.48) \\
* *\end{array}$ & $\begin{array}{l}1.00 \\
---- \\
-----\end{array}$ & & & & & & & \\
\hline во & $\begin{array}{l}0.12 \\
(6.18) \\
* * *\end{array}$ & $\begin{array}{l}0.02 \\
(0.86)\end{array}$ & $\begin{array}{l}-0.03 \\
(-1.73) \\
*\end{array}$ & $\begin{array}{l}-0.05 \\
(-2.46) \\
* *\end{array}$ & $\begin{array}{l}-0.08 \\
(-4.11) \\
* * *\end{array}$ & $\begin{array}{l}1.00 \\
---- \\
----\end{array}$ & & & & & & \\
\hline BP & $\begin{array}{l}0.02 \\
(1.21)\end{array}$ & $\begin{array}{l}0.00 \\
(0.10)\end{array}$ & $\begin{array}{l}0.01 \\
(0.48)\end{array}$ & $\begin{array}{l}0.01 \\
(0.63)\end{array}$ & $\begin{array}{l}0.04 \\
(2.11) \\
* *\end{array}$ & $\begin{array}{l}-0.01 \\
(-0.36)\end{array}$ & $\begin{array}{l}1.00 \\
---- \\
----\end{array}$ & & & & & \\
\hline PL & $\begin{array}{l}-0.09 \\
(-4.46) \\
* * *\end{array}$ & $\begin{array}{l}-0.03 \\
(-1.33)\end{array}$ & $\begin{array}{l}-0.01 \\
(-0.31)\end{array}$ & $\begin{array}{l}-0.03 \\
(-1.65) \\
*\end{array}$ & $\begin{array}{l}0.05 \\
(2.46) \\
* *\end{array}$ & $\begin{array}{l}-0.07 \\
(-3.65) \\
* * *\end{array}$ & $\begin{array}{l}-0.01 \\
(-0.64)\end{array}$ & $\begin{array}{l}1.00 \\
---- \\
----\end{array}$ & & & & \\
\hline SC & $\begin{array}{l}0.00 \\
(0.19)\end{array}$ & $\begin{array}{l}-0.21 \\
(- \\
10.78) \\
* * *\end{array}$ & $\begin{array}{l}-0.03 \\
(-1.78) \\
*\end{array}$ & $\begin{array}{l}-0.02 \\
(-1.04)\end{array}$ & $\begin{array}{l}0.34 \\
(18.31) \\
* * *\end{array}$ & $\begin{array}{l}-0.04 \\
(-2.03) \\
* *\end{array}$ & $\begin{array}{l}0.20 \\
(10.36) \\
* * *\end{array}$ & $\begin{array}{l}0.25 \\
(13.06) \\
* * *\end{array}$ & $\begin{array}{c}1.00 \\
---- \\
-----\end{array}$ & & & \\
\hline DA & $\begin{array}{l}-0.21 \\
(- \\
11.00) \\
* * *\end{array}$ & $\begin{array}{l}-0.21 \\
(- \\
11.13) \\
* * *\end{array}$ & $\begin{array}{l}-0.01 \\
(-0.42)\end{array}$ & $\begin{array}{l}-0.01 \\
(-0.58)\end{array}$ & $\begin{array}{l}0.10 \\
(5.32) \\
* * *\end{array}$ & $\begin{array}{l}-0.10 \\
(-5.13) \\
* * *\end{array}$ & $\begin{array}{l}0.02 \\
(0.83)\end{array}$ & $\begin{array}{l}0.14 \\
(7.37) \\
* * *\end{array}$ & $\begin{array}{l}0.22 \\
(11.58) \\
* * *\end{array}$ & $\begin{array}{c}1.00 \\
---- \\
----\end{array}$ & & \\
\hline AG & $\begin{array}{l}-0.14 \\
(-7.17) \\
* * *\end{array}$ & $\begin{array}{l}-0.07 \\
(-3.76) \\
* * *\end{array}$ & $\begin{array}{l}-0.06 \\
(-3.11) \\
* * *\end{array}$ & $\begin{array}{l}-0.17 \\
(-8.86) \\
* * *\end{array}$ & $\begin{array}{l}0.00 \\
(0.02)\end{array}$ & $\begin{array}{l}0.07 \\
(3.36) \\
* * *\end{array}$ & $\begin{array}{l}-0.05 \\
(-2.52) \\
* *\end{array}$ & $\begin{array}{l}0.06 \\
(3.33) \\
* * *\end{array}$ & $\begin{array}{l}0.28 \\
(14.90) \\
* * *\end{array}$ & $\begin{array}{l}0.03 \\
(1.53)\end{array}$ & $\begin{array}{l}1.00 \\
---- \\
----\end{array}$ & \\
\hline FS & 0.00 & 0.06 & -0.05 & -0.04 & -0.05 & 0.18 & -0.02 & 0.00 & 0.02 & 0.06 & 0.17 & 1.00 \\
\hline
\end{tabular}

Copyright (C) 2020, Journal of Advanced Research in Economics and Administrative Sciences (JAREAS), Under a 


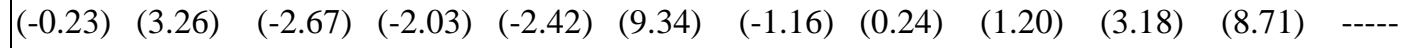
*** $* * * \quad * * \quad * * \quad * * * \quad * * * \quad * * * \quad---$

First behavior Correlation, second behavior t-Statistic first behavior significance

Table7. Midstream Industry Correlation Coefficient Matrix

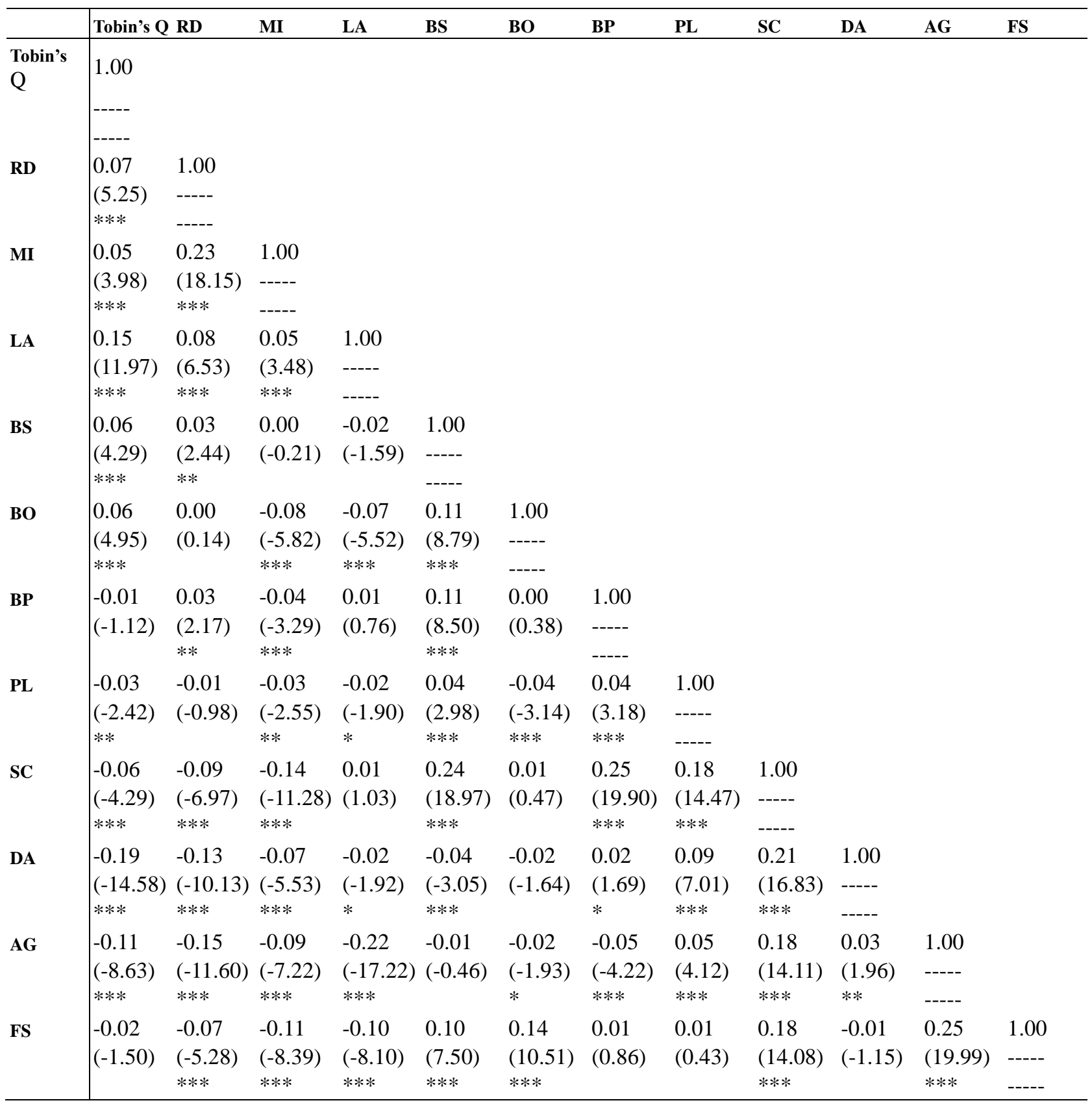

Table8. Downstream Industry Correlation Coefficient Matrix

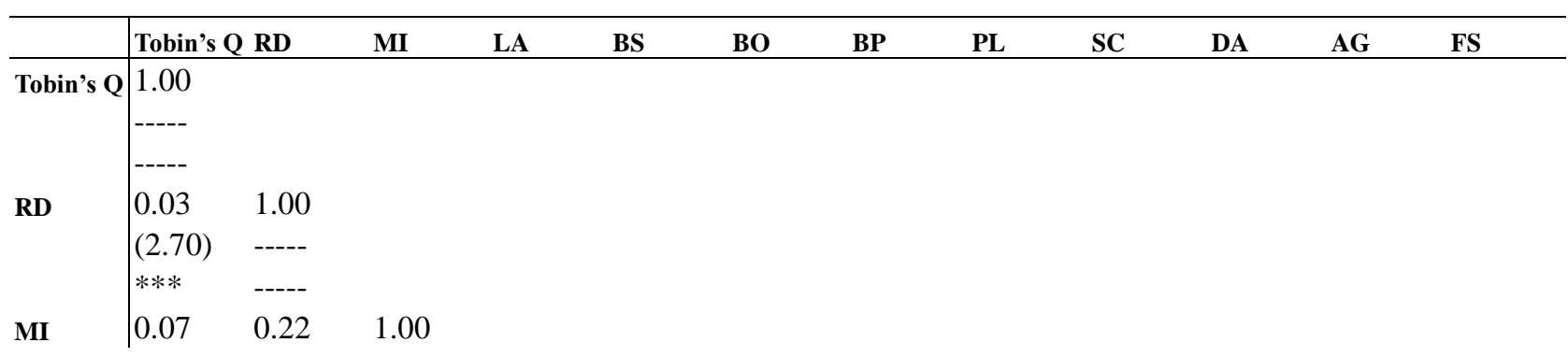

Copyright (C) 2020, Journal of Advanced Research in Economics and Administrative Sciences (JAREAS), Under a 


\begin{tabular}{|c|c|c|c|c|c|c|c|c|c|c|c|c|}
\hline & $\begin{array}{l}(5.46) \\
* * *\end{array}$ & $\begin{array}{l}(18.25) \\
* * *\end{array}$ & ----- & & & & & & & & & \\
\hline LA & $\begin{array}{l}0.05 \\
(4.14) \\
* * *\end{array}$ & $\begin{array}{l}0.02 \\
(2.02) \\
* *\end{array}$ & $\begin{array}{l}0.05 \\
(3.76) \\
* * *\end{array}$ & $\begin{array}{l}1.00 \\
---- \\
----\end{array}$ & & & & & & & & \\
\hline BS & $\begin{array}{l}0.01 \\
(0.85)\end{array}$ & $\begin{array}{l}0.00 \\
(-0.17)\end{array}$ & $\begin{array}{l}0.00 \\
(-0.08)\end{array}$ & $\begin{array}{l}-0.06 \\
(-4.57) \\
* * *\end{array}$ & $\begin{array}{l}1.00 \\
---- \\
----\end{array}$ & & & & & & & \\
\hline Во & $\begin{array}{l}0.12 \\
(9.79) \\
* * *\end{array}$ & $\begin{array}{l}-0.04 \\
(-3.05) \\
* * *\end{array}$ & $\begin{array}{l}-0.04 \\
(-3.62) \\
* * *\end{array}$ & $\begin{array}{l}-0.06 \\
(-5.05) \\
* * *\end{array}$ & $\begin{array}{l}0.10 \\
(7.98) \\
* * *\end{array}$ & $\begin{array}{c}1.00 \\
---- \\
-----\end{array}$ & & & & & & \\
\hline BP & $\begin{array}{l}-0.03 \\
(-2.58) \\
* * *\end{array}$ & $\begin{array}{l}0.00 \\
(-0.39)\end{array}$ & $\begin{array}{l}-0.01 \\
(-0.62)\end{array}$ & $\begin{array}{l}-0.01 \\
(-1.01)\end{array}$ & $\begin{array}{l}0.03 \\
(2.69) \\
* * *\end{array}$ & $\begin{array}{l}0.04 \\
(3.11) \\
* * *\end{array}$ & $\begin{array}{c}1.00 \\
----\end{array}$ & & & & & \\
\hline PL & $\begin{array}{l}-0.05 \\
(-3.70) \\
* * *\end{array}$ & $\begin{array}{l}0.02 \\
(1.95) \\
* *\end{array}$ & $\begin{array}{l}0.00 \\
(-0.09)\end{array}$ & $\begin{array}{l}-0.03 \\
(-2.69) \\
* * *\end{array}$ & $\begin{array}{l}0.04 \\
(3.24) \\
* * *\end{array}$ & $\begin{array}{l}-0.07 \\
(-5.58) \\
* * *\end{array}$ & $\begin{array}{l}0.03 \\
(2.20) \\
* *\end{array}$ & $\begin{array}{l}1.00 \\
---- \\
----\end{array}$ & & & & \\
\hline SC & $\begin{array}{l}-0.01 \\
(-1.08)\end{array}$ & $\begin{array}{l}-0.17 \\
(-13.71) \\
* * *\end{array}$ & $\begin{array}{l}-0.09 \\
(-7.48) \\
* * *\end{array}$ & $\begin{array}{l}-0.02 \\
(-1.64)\end{array}$ & $\begin{array}{l}0.25 \\
(20.89) \\
* * *\end{array}$ & $\begin{array}{l}0.07 \\
(5.95) \\
* * *\end{array}$ & $\begin{array}{l}0.29 \\
(24.08) \\
* * *\end{array}$ & $\begin{array}{l}0.20 \\
(16.69) \\
* * *\end{array}$ & $\begin{array}{l}1.00 \\
-----\end{array}$ & & & \\
\hline DA & $\begin{array}{l}-0.25 \\
(-20.77) \\
* * *\end{array}$ & $\begin{array}{l}-0.20 \\
(-16.36) \\
* * *\end{array}$ & $\begin{array}{l}-0.15 \\
(-12.14) \\
* * *\end{array}$ & $\begin{array}{l}0.01 \\
(0.74)\end{array}$ & $\begin{array}{l}-0.05 \\
(-4.38) \\
* * *\end{array}$ & $\begin{array}{l}0.04 \\
(2.91) \\
* * *\end{array}$ & $\begin{array}{l}0.04 \\
(3.05) \\
* * *\end{array}$ & $\begin{array}{l}0.08 \\
(6.77) \\
* * *\end{array}$ & $\begin{array}{l}0.26 \\
(21.50) \\
* * *\end{array}$ & $\begin{array}{l}1.00 \\
----- \\
-----\end{array}$ & & \\
\hline AG & $\begin{array}{l}-0.08 \\
(-6.54) \\
* * *\end{array}$ & $\begin{array}{l}-0.09 \\
(-7.46) \\
* * *\end{array}$ & $\begin{array}{l}-0.13 \\
(-10.81) \\
* * *\end{array}$ & $\begin{array}{l}-0.17 \\
(-14.01) \\
* * *\end{array}$ & $\begin{array}{l}0.04 \\
(3.10) \\
* * *\end{array}$ & $\begin{array}{l}0.06 \\
(5.07) \\
* * *\end{array}$ & $\begin{array}{l}-0.04 \\
(-3.48) \\
* * *\end{array}$ & $\begin{array}{l}0.07 \\
(5.41) \\
* * *\end{array}$ & $\begin{array}{l}0.22 \\
(18.37) \\
* * *\end{array}$ & $\begin{array}{l}0.07 \\
(5.46) \\
* * *\end{array}$ & $\begin{array}{c}1.00 \\
----\end{array}$ & \\
\hline FS & $\begin{array}{l}0.03 \\
(2.65) \\
* * *\end{array}$ & $\begin{array}{l}-0.01 \\
(-0.76)\end{array}$ & $\begin{array}{l}-0.10 \\
(-8.02) \\
* * *\end{array}$ & $\begin{array}{l}-0.06 \\
(-5.12) \\
* * *\end{array}$ & $\begin{array}{l}0.00 \\
(0.05)\end{array}$ & $\begin{array}{l}0.17 \\
(14.06) \\
* * *\end{array}$ & $\begin{array}{l}0.08 \\
(6.31) \\
* * *\end{array}$ & $\begin{array}{l}0.02 \\
(1.97) \\
* *\end{array}$ & $\begin{array}{l}0.22 \\
(18.57) \\
* * *\end{array}$ & $\begin{array}{l}0.01 \\
(0.99)\end{array}$ & $\begin{array}{l}0.15 \\
(12.53) \\
* * *\end{array}$ & $\begin{array}{l}1.00 \\
---- \\
----\end{array}$ \\
\hline
\end{tabular}

\section{Regression Analysis}

Before the panel data analysis is performed, a general regression analysis is performed to determine which model the sample data is suitable, and the general regression analysis model of this study is to explain the specific assets, the degree of internationalization, the structure of the board of directors and the regression analysis of other variables. After the analysis of the whole variable, this study mainly analyzes the situation of the industrial chain of the electronic industry in Taiwan, so that the industrial chain is divided into the upper and the middle, and the results of the downstream analysis are shown in Table 9-11.

Table9. Upstream Industry General Regression Model

\begin{tabular}{|c|c|c|c|c|c|c|c|c|}
\hline \multirow[t]{3}{*}{ Variable } & \multicolumn{8}{|c|}{ Coefficient、Std. Error and Significance level } \\
\hline & 1.42 & & 1.59 & & 0.83 & & 0.64 & \\
\hline & $(0.03)$ & $* * *$ & $(0.13)$ & $* * *$ & $(0.25)$ & $* * *$ & $(0.29)$ & $* *$ \\
\hline \multirow{2}{*}{ RD } & 0.39 & & & & & & 0.10 & \\
\hline & $(0.15)$ & $* *$ & & & & & $(0.15)$ & \\
\hline \multirow{2}{*}{ MI } & 28.19 & & & & & & 29.79 & \\
\hline & $(8.29)$ & $* * *$ & & & & & $(7.96)$ & $* * *$ \\
\hline \multirow{2}{*}{ LA } & 0.19 & & & & & & 0.10 & \\
\hline & $(0.05)$ & $* * *$ & & & & & $(0.05)$ & $*$ \\
\hline RD*LA & 0.27 & & & & & & 0.41 & \\
\hline
\end{tabular}

Copyright (C) 2020, Journal of Advanced Research in Economics and Administrative Sciences (JAREAS), Under a 


\begin{tabular}{|c|c|c|c|c|c|c|c|}
\hline & $(0.19)$ & & & & & $(0.19)$ & $* *$ \\
\hline \multirow{2}{*}{ RD*MI } & -11.88 & & & & & -9.38 & \\
\hline & $(6.18)$ & $*$ & & & & $(5.96)$ & \\
\hline \multirow{2}{*}{ MI*LA } & -14.01 & & & & & -14.04 & \\
\hline & $(8.43)$ & $*$ & & & & (8.09) & $*$ \\
\hline \multirow{2}{*}{ RD*MI*LA } & 32.55 & & & & & 26.51 & \\
\hline & $(16.84)$ & $*$ & & & & (16.17) & \\
\hline \multirow{2}{*}{ FS } & & -9.89 & & & & -12.92 & \\
\hline & & $(4.27)$ & $* *$ & & & $(4.08)$ & $* * *$ \\
\hline \multirow{2}{*}{$\mathrm{FS}^{2}$} & & 98.77 & & & & 131.33 & \\
\hline & & (40.64) & $* *$ & & & (38.79) & $* * *$ \\
\hline \multirow[b]{2}{*}{$\mathbf{F S}^{3}$} & & -356.76 & & & & -485.65 & \\
\hline & & $(157.85)$ & $* *$ & & & $(150.53)$ & $* * *$ \\
\hline \multirow[b]{2}{*}{$\mathbf{F S}^{4}$} & & 605.85 & & & & 840.37 & \\
\hline & & $(290.83)$ & $* *$ & & & $(277.23)$ & $* * *$ \\
\hline \multirow{2}{*}{$\mathbf{F S}^{5}$} & & -494.04 & & & & -693.24 & \\
\hline & & $(253.58)$ & $*$ & & & $(241.67)$ & $* * *$ \\
\hline \multirow{2}{*}{$\mathrm{FS}^{6}$} & & 156.27 & & & & 220.42 & \\
\hline & & $(84.25)$ & $*$ & & & $(80.28)$ & $* * *$ \\
\hline \multirow[b]{2}{*}{ BS } & & & & 0.01 & & 0.02 & \\
\hline & & & & $(0.01)$ & & $(0.01)$ & \\
\hline \multirow{2}{*}{ BO } & & & & 0.94 & & 0.95 & \\
\hline & & & & $(0.16)$ & $* * *$ & $(0.16)$ & $* * *$ \\
\hline \multirow{2}{*}{ BP } & & & & -0.02 & & -0.02 & \\
\hline & & & & $(0.07)$ & & $(0.07)$ & \\
\hline \multirow{2}{*}{ PL } & & & & -0.66 & & -0.70 & \\
\hline & & & & $(0.19)$ & $* * *$ & $(0.19)$ & $* * *$ \\
\hline \multirow{2}{*}{ SC } & & & & 0.09 & & 0.10 & \\
\hline & & & & $(0.02)$ & $* * *$ & $(0.02)$ & $* * *$ \\
\hline \multirow{2}{*}{ DA } & & & & -1.59 & & -1.56 & \\
\hline & & & & $(0.15)$ & $* * *$ & $(0.15)$ & $* * *$ \\
\hline \multirow[b]{2}{*}{ AG } & & & & -0.03 & & -0.03 & \\
\hline & & & & $(0.00)$ & $* * *$ & $(0.00)$ & $* * *$ \\
\hline R2 & 0.03 & 0.01 & & 0.09 & & 0.11 & \\
\hline Adjusted R2 & 0.02 & 0.00 & & 0.08 & & 0.11 & \\
\hline F-statistic & 9.62 & 2.84 & & 35.71 & & 16.44 & \\
\hline Prob & 0.00 & 0.01 & & 0.00 & & 0.00 & \\
\hline
\end{tabular}

Table10. Midstream Industry General regression model

\begin{tabular}{|c|c|c|c|c|c|c|c|c|}
\hline Variable & \multicolumn{8}{|c|}{ Coefficient、Std. Error and Significance level } \\
\hline \multirow{2}{*}{$\mathbf{C}$} & \multicolumn{2}{|l|}{0.98} & \multicolumn{2}{|l|}{1.16} & \multicolumn{2}{|l|}{1.42} & \multicolumn{2}{|l|}{1.37} \\
\hline & $(0.01)$ & $* * *$ & $(0.05)$ & $* * *$ & $(0.12)$ & $* * *$ & $(0.13)$ & $* * *$ \\
\hline \multirow{2}{*}{ RD } & 1.24 & & & & & & 0.76 & \\
\hline & $(0.19)$ & $* * *$ & & & & & $(0.19)$ & $* * *$ \\
\hline \multirow{2}{*}{ MI } & 7.52 & & & & & & 5.66 & \\
\hline & $(2.45)$ & $* * *$ & & & & & $(2.44)$ & $* *$ \\
\hline \multirow{2}{*}{$\mathbf{L A}$} & 0.30 & & & & & & 0.28 & \\
\hline & $(0.02)$ & $* * *$ & & & & & $(0.02)$ & $* * *$ \\
\hline RD*LA & -0.52 & & & & & & -0.42 & \\
\hline
\end{tabular}




\begin{tabular}{|c|c|c|c|c|c|c|}
\hline & $(0.10)$ & $* * *$ & & & $(0.10)$ & $* * *$ \\
\hline \multirow{2}{*}{ RD*MI } & -18.51 & & & & -10.76 & \\
\hline & $(10.97)$ & $*$ & & & (10.77) & \\
\hline \multirow{2}{*}{ MI*LA } & -8.09 & & & & -10.74 & \\
\hline & $(4.00)$ & $* *$ & & & $(3.92)$ & $* * *$ \\
\hline \multirow{2}{*}{ RD*MI*LA } & 6.48 & & & & 5.36 & \\
\hline & $(5.73)$ & & & & $(5.62)$ & \\
\hline \multirow{2}{*}{ FS } & & -3.66 & & & -4.19 & \\
\hline & & $(1.23)$ & $* * *$ & & (1.19) & $* * *$ \\
\hline \multirow{2}{*}{$\mathbf{F S}^{2}$} & & 24.20 & & & 26.79 & \\
\hline & & $(8.06)$ & $* * *$ & & $(7.80)$ & $* * *$ \\
\hline \multirow{2}{*}{$\mathbf{F S}^{3}$} & & -58.62 & & & -63.94 & \\
\hline & & (20.16) & $* * *$ & & $(19.46)$ & $* * *$ \\
\hline \multirow{2}{*}{$\mathbf{F S}^{4}$} & & 60.28 & & & 65.23 & \\
\hline & & $(21.50)$ & $* * *$ & & $(20.75)$ & $* * *$ \\
\hline \multirow{2}{*}{$\mathbf{F S}^{5}$} & & -22.37 & & & -24.02 & \\
\hline & & $(8.24)$ & $* * *$ & & $(7.95)$ & $* * *$ \\
\hline \multirow{2}{*}{ BS } & & & 0.02 & & 0.02 & \\
\hline & & & $(0.01)$ & $* * *$ & $(0.01)$ & $* * *$ \\
\hline \multirow{2}{*}{ BO } & & & 0.28 & & 0.35 & \\
\hline & & & $(0.07)$ & $* * *$ & $(0.07)$ & $* * *$ \\
\hline \multirow{2}{*}{ BP } & & & -0.06 & & -0.05 & \\
\hline & & & $(0.04)$ & & $(0.04)$ & \\
\hline \multirow{2}{*}{ PL } & & & -0.04 & & -0.04 & \\
\hline & & & $(0.07)$ & & $(0.07)$ & \\
\hline \multirow{2}{*}{ SC } & & & 0.00 & & 0.00 & \\
\hline & & & $(0.01)$ & & $(0.01)$ & \\
\hline \multirow{2}{*}{ DA } & & & -0.90 & & -0.85 & \\
\hline & & & $(0.07)$ & $* * *$ & $(0.07)$ & $* * *$ \\
\hline \multirow{2}{*}{ AG } & & & -0.01 & & -0.01 & \\
\hline & & & $(0.00)$ & $* * *$ & $(0.00)$ & $* * *$ \\
\hline $\mathbf{R}^{2}$ & 0.00 & 0.05 & 0.08 & & 0.03 & \\
\hline Adjusted $\mathbf{R}^{2}$ & 0.00 & 0.05 & 0.07 & & 0.03 & \\
\hline F-statistic & 2.71 & 46.01 & 26.27 & & 30.66 & \\
\hline Prob & 0.02 & 0.00 & 0.00 & & 0.00 & \\
\hline
\end{tabular}

Table11. Downstream Industry General regression model

\begin{tabular}{|c|c|c|c|c|c|c|c|c|}
\hline \multirow[t]{3}{*}{ Variable } & \multicolumn{8}{|c|}{ Coefficient、Std. Error and Significance level } \\
\hline & 1.06 & & 1.04 & & 1.05 & & 0.96 & \\
\hline & $(0.01)$ & $* * *$ & $(0.03)$ & $* * *$ & $(0.11)$ & $* * *$ & $(0.12)$ & $* * *$ \\
\hline \multirow{2}{*}{ RD } & 0.33 & & & & & & -0.04 & \\
\hline & $(0.10)$ & $* * *$ & & & & & $(0.10)$ & \\
\hline MI & 7.52 & & & & & & 3.68 & \\
\hline
\end{tabular}




\begin{tabular}{|c|c|c|c|c|c|c|c|}
\hline & (1.53) & $* * *$ & & & & (1.49) & $* *$ \\
\hline \multirow{2}{*}{ LA } & 0.09 & & & & & 0.08 & \\
\hline & $(0.02)$ & $* * *$ & & & & $(0.02)$ & $* * *$ \\
\hline \multirow{2}{*}{ RD*LA } & -0.12 & & & & & -0.06 & \\
\hline & $(0.12)$ & & & & & $(0.12)$ & \\
\hline \multirow{2}{*}{ RD*MI } & -2.78 & & & & & 2.32 & \\
\hline & (4.13) & & & & & (3.97) & \\
\hline \multirow{2}{*}{ MI*LA } & -1.83 & & & & & -1.38 & \\
\hline & $(1.90)$ & & & & & $(1.82)$ & \\
\hline \multirow{2}{*}{ RD*MI*LA } & -1.40 & & & & & -1.80 & \\
\hline & $(3.99)$ & & & & & $(3.81)$ & \\
\hline \multirow{2}{*}{ FS } & & 1.39 & & & & 1.21 & \\
\hline & & $(0.60)$ & $* *$ & & & $(0.57)$ & $* *$ \\
\hline \multirow{2}{*}{$\mathrm{FS}^{2}$} & & -6.68 & & & & -5.51 & \\
\hline & & $(2.73)$ & $* *$ & & & $(2.61)$ & $* *$ \\
\hline \multirow{2}{*}{ FS $^{3}$} & & 10.71 & & & & 8.59 & \\
\hline & & $(4.16)$ & $* *$ & & & (3.98) & $* *$ \\
\hline \multirow{2}{*}{$\mathrm{FS}^{4}$} & & -5.37 & & & & -4.27 & \\
\hline & & $(2.03)$ & $* * *$ & & & $(1.94)$ & $* *$ \\
\hline \multirow{2}{*}{ BS } & & & & -0.02 & & -0.01 & \\
\hline & & & & $(0.01)$ & $* * *$ & $(0.01)$ & $* *$ \\
\hline \multirow{2}{*}{ BO } & & & & 0.73 & & 0.72 & \\
\hline & & & & $(0.07)$ & $* * *$ & $(0.07)$ & $* * *$ \\
\hline \multirow{2}{*}{ BP } & & & & -0.15 & & -0.15 & \\
\hline & & & & $(0.03)$ & $* * *$ & $(0.03)$ & $* * *$ \\
\hline \multirow{2}{*}{$\mathbf{P L}$} & & & & -0.15 & & -0.14 & \\
\hline & & & & $(0.08)$ & $* *$ & $(0.08)$ & $*$ \\
\hline \multirow{2}{*}{ SC } & & & & 0.06 & & 0.06 & \\
\hline & & & & $(0.01)$ & $* * *$ & $(0.01)$ & $* * *$ \\
\hline \multirow{2}{*}{ DA } & & & & -1.31 & & -1.30 & \\
\hline & & & & $(0.06)$ & $* * *$ & $(0.06)$ & $* * *$ \\
\hline \multirow{2}{*}{ AG } & & & & -0.01 & & -0.01 & \\
\hline & & & & $(0.00)$ & $* * *$ & $(0.00)$ & $* * *$ \\
\hline $\mathbf{R} 2$ & 0.01 & 0.00 & & 0.09 & & 0.10 & \\
\hline Adjusted R2 & 0.01 & 0.00 & & 0.09 & & 0.09 & \\
\hline F-statistic & 8.53 & 3.73 & & 94.23 & & 38.65 & \\
\hline Prob & 0.00 & 0.00 & & 0.00 & & 0.00 & \\
\hline
\end{tabular}

From Table 9 to 11, 12 regression equations are constructed in this study. Through $\mathrm{F}$ test, the 12 regression equations can be obtained, which indicates that all variables have explanatory power to corporate performance.

The overall regression equations of the upper, middle and lower reaches are constructed as follows:

\section{Model 1.Upstream global regression equation}


Tobin's $\quad \mathrm{Q}=0.64+0.10 \mathrm{RD}+29.79 \mathrm{MI}+0.10 \mathrm{LA}+0.41 \mathrm{RD} * \mathrm{LA}-9.38 \mathrm{RD} * \mathrm{MI}-14.04 \mathrm{MI} * \mathrm{LA}+$ 26.51RD*MI*LA

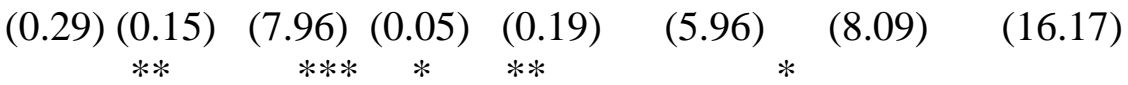

$$
\begin{aligned}
& \text {-12.92FS +131.33FS2 -485.65FS3 +840.37FS4-693.24FS5 +220.42FS6 } \\
& \begin{array}{cccccc}
(4.08) & (38.79) & (150.53) & (277.23) & (241.67) & (80.28) \\
* * * & * * * & * * * & * * * & * * * & * * *
\end{array} \\
& +0.02 \mathrm{BS}+0.95 \mathrm{BO}-0.02 \mathrm{BP}-0.70 \mathrm{PL}+0.10 \mathrm{SC}-1.56 \mathrm{DA}-0.03 \mathrm{AG} \\
& \begin{array}{llllll}
(0.01)(0.16) & (0.07)(0.19)(0.02)(0.15) & (0.00)
\end{array}
\end{aligned}
$$

Model 2.Middle reaches global regression equation

Tobin's $\quad \mathrm{Q}=1.37+0.76 \mathrm{RD}+5.66 \mathrm{MI}+0.28 \mathrm{LA}-0.42 \mathrm{RD} * \mathrm{LA}-10.76 \mathrm{RD} * \mathrm{MI}-10.74 \mathrm{MI} * \mathrm{LA}+$ 5.36RD*MI*LA

$$
\begin{aligned}
& \begin{array}{cccccc}
(0.13) & (0.19) & (2.44) & (0.02) & (0.10) & (10.77) \\
* * * & * * * & * * & * * * & * * * & * * *
\end{array} \\
& \text {-4.19FS +26.79FS2 -63.94FS3 +65.23FS4-24.02FS5 }
\end{aligned}
$$

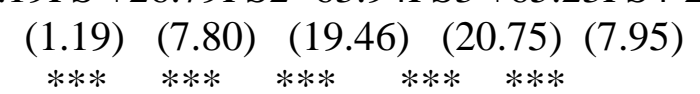

$$
\begin{aligned}
& +0.02 \mathrm{BS}+0.35 \mathrm{BO}-0.05 \mathrm{BP}-0.04 \mathrm{PL}+0.00 \mathrm{SC}-0.85 \mathrm{DA}-0.01 \mathrm{AG}
\end{aligned}
$$

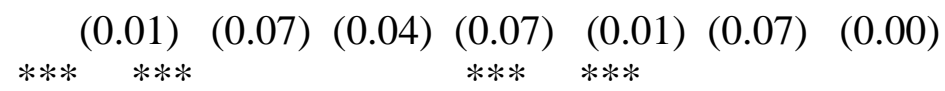

Model 3.Downstream global regression equation

Tobin's $\quad \mathrm{Q}=0.96-0.04 \mathrm{RD}+3.68 \mathrm{MI}+0.08 \mathrm{LA}-0.06 \mathrm{RD} * \mathrm{LA}-2.32 \mathrm{RD} * \mathrm{MI}-1.38 \mathrm{MI}$ LA1.80RD*MI*LA

$$
\begin{aligned}
& \begin{array}{ccccc}
(0.12) & (0.10) & (1.49) & (0.02) & (0.12) \\
* * * & * * & * * * & (3.97)
\end{array} \\
& -0.01 \mathrm{BS}+0.72 \mathrm{BO}-0.15 \mathrm{BP}-0.14 \mathrm{PL}+0.06 \mathrm{SC}-1.30 \mathrm{DA}-0.01 \mathrm{AG} \\
& \text { (0.01) (0.07) (0.03) (0.08) (0.01) (0.06) (0.00) } \\
& * * \quad * * * \quad * * * \quad * \quad * * * \quad * * * \quad * * * \\
& \text { 1.21FS -5.51FS2 +8.59FS3 -4.27FS4 } \\
& \text { (0.57) (2.61) (3.98) (1.94) }
\end{aligned}
$$

According to Table 9 11 and Models 1 3, it is preliminarily determined that the impact of exclusive assets on the company's performance has some interaction. The internationalization degree affects the $\mathrm{W}$-shaped influence on the downstream industry of the company's performance electronics industry, while the upper and middle reaches the middle reaches the higher order, and other control variables have significant influence on the company performance.

\section{Panel Data Analysis}

The sample of this study is Panel Data, so it is necessary to judge whether the time series and cross-section data of the sample data have influence. In this study, the mixed regression model (Pooled Regression Model) is used to judge, and the correlation analysis results are as follows: table 12 / 14.

Table 12.Upstream Industry Pool Regression Equation Model

\begin{tabular}{lllllllll} 
Variable & \multicolumn{7}{l}{ Coefficient、 Std. Error and Significance level } \\
\hline $\mathbf{C}$ & 1.24 & 1.42 & 0.61 & 0.58 & \\
\cline { 2 - 9 } & $(0.02)$ & $* * *$ & $(0.06)$ & $* * *$ & $(0.12)$ & $* * *$ & $(0.14)$ & $* * *$ \\
\hline
\end{tabular}




\begin{tabular}{|c|c|c|c|c|c|c|}
\hline \multirow[b]{2}{*}{ RD } & \multicolumn{4}{|l|}{0.16} & \multicolumn{2}{|l|}{0.00} \\
\hline & \multicolumn{4}{|l|}{$(0.10)$} & \multicolumn{2}{|l|}{$(0.09)$} \\
\hline \multirow{2}{*}{ MI } & 4.17 & & & & 13.44 & \\
\hline & $(5.96)$ & & & & $(5.68)$ & $* *$ \\
\hline \multirow[b]{2}{*}{ LA } & 0.14 & & & & 0.11 & \\
\hline & $(0.03)$ & $* * *$ & & & $(0.03)$ & $* * *$ \\
\hline \multirow{2}{*}{ RD*LA } & -0.05 & & & & 0.02 & \\
\hline & $(0.14)$ & & & & $(0.14)$ & \\
\hline \multirow[b]{2}{*}{ RD*MI } & -2.21 & & & & -4.77 & \\
\hline & $(9.85)$ & & & & $(9.93)$ & \\
\hline \multirow{2}{*}{ MI*LA } & -2.79 & & & & -6.09 & \\
\hline & $(5.22)$ & & & & $(5.19)$ & \\
\hline \multirow{2}{*}{ RD*MI* LA } & 0.78 & & & & -1.57 & \\
\hline & $(19.82)$ & & & & $(19.51)$ & \\
\hline \multirow{2}{*}{ FS } & & -9.33 & & & -7.04 & \\
\hline & & $(1.99)$ & $* * *$ & & $(1.82)$ & $* * *$ \\
\hline \multirow{2}{*}{$\mathrm{FS}^{2}$} & & 82.73 & & & 65.02 & \\
\hline & & $(19.17)$ & $* * *$ & & $(17.61)$ & $* * *$ \\
\hline \multirow{2}{*}{$\mathbf{F S}^{3}$} & & -293.45 & & & -234.39 & \\
\hline & & $(75.13)$ & $* * *$ & & $(69.49)$ & $* * *$ \\
\hline \multirow{2}{*}{$\mathrm{FS}^{4}$} & & 506.42 & & & 407.54 & \\
\hline & & $(139.56)$ & $* * *$ & & $(130.01)$ & $* * *$ \\
\hline \multirow{2}{*}{$\mathbf{F S}^{5}$} & & -422.57 & & & -342.60 & \\
\hline & & $(122.68)$ & $* * *$ & & $(114.95)$ & $* * *$ \\
\hline \multirow{2}{*}{$\mathrm{FS}^{6}$} & & 136.23 & & & 111.69 & \\
\hline & & $(41.09)$ & $* * *$ & & $(38.68)$ & $* * *$ \\
\hline \multirow[b]{2}{*}{ BS } & & & & 0.01 & 0.02 & \\
\hline & & & & $(0.01) \quad *$ & $(0.01)$ & $* *$ \\
\hline \multirow{2}{*}{ BO } & & & & 0.60 & 0.61 & \\
\hline & & & & $(0.08) \quad * * *$ & $(0.08)$ & $* * *$ \\
\hline \multirow{2}{*}{ BP } & & & & -0.11 & -0.12 & \\
\hline & & & & $(0.04)$ & $(0.04)$ & $* * *$ \\
\hline \multirow{2}{*}{ PL } & & & & -0.26 & -0.28 & \\
\hline & & & & $(0.09) \quad * * *$ & $(0.10)$ & $* * *$ \\
\hline \multirow{2}{*}{ SC } & & & & 0.07 & 0.07 & \\
\hline & & & & $(0.01)$ & $(0.01)$ & $* * *$ \\
\hline \multirow{2}{*}{ DA } & & & & -0.95 & -0.99 & \\
\hline & & & & $(0.07) \quad * * *$ & $(0.07)$ & $* * *$ \\
\hline \multirow{2}{*}{ AG } & & & & -0.02 & -0.02 & \\
\hline & & & & $(0.00) \quad * * *$ & $(0.00)$ & $* * *$ \\
\hline Weighted Statistics R2 & 0.01 & 0.02 & & 0.15 & 0.17 & \\
\hline $\begin{array}{ll}\text { Weighted } & \text { Statistics } \\
\text { SSE } & \\
\end{array}$ & $\mathbf{s}_{3983.27}$ & 4092.23 & & 3738.88 & 3617.65 & \\
\hline $\begin{array}{ll}\text { Unweighted Statistics } \\
\text { R2 }\end{array}$ & -0.02 & -0.03 & & 0.04 & 0.06 & \\
\hline $\begin{array}{ll}\text { Unweighted Statistics } \\
\text { SSE }\end{array}$ & $\mathbf{s}_{4814.74}$ & 4842.71 & & 4510.57 & 4434.80 & \\
\hline
\end{tabular}


The upstream of the electronics industry is found in Table 12 that the weighted R-squared of the four groups $(0.01,0.02,0.15 \& 0.17)$ is larger than the unweighted R2 $(-0.020 .030 .004 \& 0.006)$ and the weighted Sum squared reside $(3983.27,4092.23,3738.88 \& 3617.65)$ is also higher than the unweighted Sum squared reside $(4814.74,4842.71,4510.57 \& 4434.80)$ This result indicates that the sample upstream of the study electronics industry is suitable for Panel Data Analysis.

Table13. Midstream Industry Pool Regression Equation Model

\begin{tabular}{|c|c|c|c|c|c|c|c|c|}
\hline \multirow{3}{*}{$\begin{array}{l}\text { Variable } \\
\mathrm{C}\end{array}$} & \multirow{2}{*}{\multicolumn{2}{|c|}{$\begin{array}{l}\text { Coefficient、Std } \\
0.91 \\
\end{array}$}} & \multicolumn{3}{|c|}{ Std. Error and Significance level } & \multirow{2}{*}{\multicolumn{2}{|c|}{1.26}} & \multirow[b]{3}{*}{$* * *$} \\
\hline & & & 1.03 & & 1.23 & & & \\
\hline & $(0.01)$ & $* * *$ & $(0.02)$ & $* * *$ & $(0.06)$ & $* * *$ & $(0.06)$ & \\
\hline \multirow[b]{2}{*}{ RD } & 0.44 & & & & & & 0.21 & \\
\hline & $(0.13)$ & $* * *$ & & & & & $(0.12)$ & $*$ \\
\hline \multirow{2}{*}{ MI } & 5.52 & & & & & & 2.94 & \\
\hline & $(1.49)$ & $* * *$ & & & & & $(1.64)$ & $*$ \\
\hline \multirow[b]{2}{*}{ LA } & 0.16 & & & & & & 0.18 & \\
\hline & $(0.01)$ & $* * *$ & & & & & $(0.01)$ & $* * *$ \\
\hline \multirow[b]{2}{*}{ RD*LA } & -0.18 & & & & & & -0.16 & \\
\hline & $(0.07)$ & $* * *$ & & & & & $(0.07)$ & $* *$ \\
\hline \multirow{2}{*}{ RD*MI } & -13.13 & & & & & & -13.22 & \\
\hline & $(9.34)$ & & & & & & $(9.65)$ & \\
\hline \multirow{2}{*}{ MI*LA } & -6.77 & & & & & & -10.61 & \\
\hline & $(2.01)$ & $* * *$ & & & & & $(2.18)$ & $* * *$ \\
\hline \multirow{2}{*}{ RD*MI*LA } & 5.41 & & & & & & 7.93 & \\
\hline & $(4.80)$ & & & & & & $(4.96)$ & \\
\hline \multirow{2}{*}{ FS } & & & -1.32 & & & & -1.74 & \\
\hline & & & $(0.57)$ & $* *$ & & & $(0.55)$ & $* * *$ \\
\hline \multirow{2}{*}{$\mathbf{F S}^{2}$} & & & 6.95 & & & & 9.50 & \\
\hline & & & $(3.73)$ & $*$ & & & $(3.64)$ & $* * *$ \\
\hline \multirow{2}{*}{$\mathrm{FS}^{3}$} & & & -14.57 & & & & -20.28 & \\
\hline & & & $(9.36)$ & & & & $(9.16)$ & $* *$ \\
\hline \multirow{2}{*}{$F^{4}$} & & & 13.34 & & & & 18.88 & \\
\hline & & & $(10.02)$ & & & & $(9.80)$ & $*$ \\
\hline \multirow{2}{*}{$\mathbf{F S}^{5}$} & & & -4.55 & & & & -6.47 & \\
\hline & & & $(3.85)$ & & & & $(3.77)$ & $*$ \\
\hline \multirow{2}{*}{ BS } & & & & & 0.01 & & 0.01 & \\
\hline & & & & & $(0.00)$ & $* * *$ & $(0.00)$ & $* * *$ \\
\hline \multirow{2}{*}{ BO } & & & & & 0.15 & & 0.17 & \\
\hline & & & & & $(0.03)$ & $* * *$ & $(0.03)$ & $* * *$ \\
\hline \multirow{2}{*}{ BP } & & & & & -0.01 & & 0.00 & \\
\hline & & & & & $(0.02)$ & & $(0.02)$ & \\
\hline \multirow{2}{*}{ PL } & & & & & -0.07 & & -0.06 & \\
\hline & & & & & $(0.03)$ & $* *$ & $(0.03)$ & $*$ \\
\hline \multirow{2}{*}{ SC } & & & & & 0.00 & & 0.00 & \\
\hline & & & & & $(0.00)$ & & $(0.00)$ & \\
\hline \multirow{2}{*}{ DA } & & & & & -0.59 & & -0.61 & \\
\hline & & & & & $(0.03)$ & $* * *$ & $(0.03)$ & $* * *$ \\
\hline & & & & & -0.01 & & -0.01 & \\
\hline AG & & & & & $(0.00)$ & $* * *$ & $(0.00)$ & $* * *$ \\
\hline Weighted Statistics R2 & 0.11 & & 0.01 & & 0.10 & & 0.12 & \\
\hline Weighted Statistics SSE & 3221.07 & & 3301.45 & & 3210.88 & & 3082.20 & \\
\hline $\begin{array}{ll}\text { Unweighted Statistics } \\
\text { R2 }\end{array}$ & 0.00 & & -0.03 & & 0.03 & & 0.05 & \\
\hline
\end{tabular}




\begin{tabular}{lllll}
\hline Unweighted & Statistics $_{3690.08}$ & 3793.11 & 3603.41 & 3530.55 \\
SSE & &
\end{tabular}

In Table 13, it is found that the weighted R2 of the four groups is larger than the unweighted R2 $(0.00,0.03,0.03 \& 3082.20)$ and the weighted Sum squared reside (3221.073301.45,3301.45, $3210.88 \& 3082.20)$ is also higher than the unweighted Sum squared reside (3690.083793.11, $3603.41 \& 3082.20)$. 0.55) this result indicates that the mid-stream sample of the electronics industry in this study is suitable for Panel Data Analysis.

Table14. Downstream Industry Pool Regression Equation Model

\begin{tabular}{|c|c|c|c|c|c|c|c|c|}
\hline Variable & \multirow{2}{*}{$\begin{array}{l}\text { Coefficient、 } \\
0.92\end{array}$} & \multicolumn{4}{|c|}{ Std. Error and Significance level } & \multirow{2}{*}{\multicolumn{2}{|c|}{0.82}} & \multirow[b]{3}{*}{$* * *$} \\
\hline \multirow{2}{*}{$\mathbf{C}$} & & & 0.92 & & 0.88 & & & \\
\hline & $(0.01)$ & $* * *$ & $(0.01)$ & $* * *$ & $(0.05)$ & $* * *$ & $(0.06)$ & \\
\hline \multirow{2}{*}{ RD } & 0.22 & & & & & & 0.00 & \\
\hline & $(0.06)$ & $* * *$ & & & & & $(0.04)$ & \\
\hline \multirow{2}{*}{ MI } & 5.75 & & & & & & 3.74 & \\
\hline & $(0.97)$ & $* * *$ & & & & & $(0.87)$ & $* * *$ \\
\hline \multirow{2}{*}{ LA } & 0.07 & & & & & & 0.07 & \\
\hline & $(0.01)$ & $* * *$ & & & & & $(0.01)$ & $* * *$ \\
\hline \multirow{2}{*}{ RD*LA } & -0.09 & & & & & & -0.04 & \\
\hline & $(0.08)$ & & & & & & $(0.06)$ & \\
\hline \multirow{2}{*}{ RD*MI } & -3.32 & & & & & & -0.52 & \\
\hline & $(3.22)$ & & & & & & $(2.68)$ & \\
\hline \multirow{2}{*}{ MI*LA } & -3.87 & & & & & & -3.24 & \\
\hline & $(1.06)$ & $* * *$ & & & & & $(1.19)$ & $* * *$ \\
\hline \multirow{2}{*}{ RD*MI*LA } & 1.10 & & & & & & 0.44 & \\
\hline & $(3.11)$ & & & & & & $(2.71)$ & \\
\hline \multirow{2}{*}{ FS } & & & 0.84 & & & & 0.68 & \\
\hline & & & $(0.24)$ & $* * *$ & & & $(0.23)$ & $* * *$ \\
\hline \multirow{2}{*}{$\mathbf{F S}^{2}$} & & & -3.12 & & & & -2.22 & \\
\hline & & & (1.17) & $* * *$ & & & (1.08) & $* *$ \\
\hline \multirow{2}{*}{$\mathrm{FS}^{3}$} & & & 4.01 & & & & 2.68 & \\
\hline & & & $(1.84)$ & $* *$ & & & $(1.67)$ & \\
\hline \multirow{2}{*}{$\mathbf{F S}^{4}$} & & & -1.68 & & & & -1.10 & \\
\hline & & & $(0.92)$ & $*$ & & & $(0.83)$ & \\
\hline \multirow{2}{*}{ BS } & & & & & 0.00 & & 0.00 & \\
\hline & & & & & $(0.00)$ & & $(0.00)$ & \\
\hline \multirow{2}{*}{ BO } & & & & & 0.44 & & 0.44 & \\
\hline & & & & & $(0.03)$ & $* * *$ & $(0.03)$ & $* * *$ \\
\hline \multirow{2}{*}{ BP } & & & & & -0.08 & & -0.06 & \\
\hline & & & & & $(0.02)$ & $* * *$ & $(0.02)$ & $* * *$ \\
\hline \multirow{2}{*}{ PL } & & & & & -0.11 & & -0.10 & \\
\hline & & & & & $(0.03)$ & $* * *$ & $(0.03)$ & $* * *$ \\
\hline \multirow{2}{*}{ SC } & & & & & 0.04 & & 0.04 & \\
\hline & & & & & $(0.00)$ & $* * *$ & $(0.00)$ & $* * *$ \\
\hline
\end{tabular}




\begin{tabular}{|c|c|c|c|c|c|c|}
\hline \multirow{2}{*}{ DA } & & & \multicolumn{2}{|l|}{-0.85} & \multicolumn{2}{|l|}{-0.86} \\
\hline & & & $(0.03)$ & $* * *$ & $(0.03)$ & $* * *$ \\
\hline \multirow{2}{*}{ AG } & & & 0.00 & & 0.00 & \\
\hline & & & $(0.00)$ & $* * *$ & $(0.00)$ & $* * *$ \\
\hline Weighted Statistics R2 & 0.01 & 0.00 & 0.15 & & 0.16 & \\
\hline Weighted Statistics SSE & 4173.32 & 4185.50 & 3926.56 & & 3893.89 & \\
\hline $\begin{array}{l}\text { Unweighted Statistics } \\
\text { R2 }\end{array}$ & s-0.03 & -0.03 & 0.06 & & 0.06 & \\
\hline $\begin{array}{l}\text { Unweighted Statistics } \\
\text { SSE }\end{array}$ & $s_{4967.53}$ & 5005.10 & 4577.68 & & 4559.41 & \\
\hline
\end{tabular}

The weighted R2 (-0.03,-0.03, $0.06 \& 0.06)$ and weighted Sum-squared reside (4173.32, $4185.50,3926.56 \& 3893.89)$, which is greater than the unweighted R2 $(-0.03,-0.03,0.06 \&$ 0.06) and weighted Sum-squared reside (4967.53, 5005.10, 4577.68 \& 4559), is found downstream of the electronic industry in Table 14. .41) This result indicates that the downstream samples of the electronic industry of this study are suitable for Panel Data Analysis.

The study samples are then determined to be suitable for Panel Data Analysis, followed by the selection of the fixed effect and the random effect model. The results of the analysis are shown in Table 15-17. The results of the analysis on the upstream of the electronic industry are shown in Table 15, and the four groups of analysis are suitable for analysis with a fixed-effect model, such as model 4-8.

Table15. Upstream Industry Fixed and Random Effects Model

\begin{tabular}{|c|c|c|c|c|c|c|c|c|c|c|c|c|}
\hline Variable & FIXED & & RANDOM & FIXED & & RANDOM & FIXED & RANDOM & FIXED & & RANDOM & \\
\hline \multirow{2}{*}{ 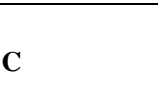 } & 1.41 & & 1.65 & 1.58 & & 2.03 & 0.89 & 0.88 & 0.63 & & 1.32 & \\
\hline & $(0.03)$ & $* * *$ & $(0.06) *$ & $* * *(0.13)$ & $* * *$ & $(0.13)$ & $* * *(0.24) * * *$ & $*(0.42) \quad * *$ & $(0.28)$ & $* *$ & $(0.42)$ & $* * *$ \\
\hline \multirow[b]{2}{*}{ RD } & 0.46 & & -0.47 & & & & & & 0.13 & & -0.40 & \\
\hline & $(0.15)$ & $* * *$ & $(0.14)$ & $* * *$ & & & & & $(0.15)$ & & $(0.14)$ & $* * *$ \\
\hline \multirow{2}{*}{ MI } & 26.57 & & 10.38 & & & & & & 26.99 & & 13.11 & \\
\hline & $(8.07)$ & $* * *$ & $(7.65)$ & & & & & & $(7.74)$ & $* * *$ & $(7.52)$ & $*$ \\
\hline \multirow[b]{2}{*}{ LA } & 0.21 & & 0.04 & & & & & & 0.12 & & -0.01 & \\
\hline & $(0.05)$ & $* * *$ & $(0.05)$ & & & & & & $(0.05)$ & $* *$ & $(0.05)$ & \\
\hline \multirow[b]{2}{*}{ RD*LA } & 0.20 & & 0.50 & & & & & & 0.36 & & 0.50 & \\
\hline & $(0.19)$ & & $(0.16)$ * & $* * *$ & & & & & $(0.18)$ & $* *$ & $(0.16)$ & $* * *$ \\
\hline \multirow{2}{*}{ RD*MI } & -11.54 & & 0.60 & & & & & & -8.42 & & -2.63 & \\
\hline & $(6.01)$ & $*$ & $(5.26)$ & & & & & & $(5.79)$ & & $(5.17)$ & \\
\hline \multirow{2}{*}{ MI*LA } & -14.85 & & -5.45 & & & & & & -14.52 & & -4.77 & \\
\hline & $(8.19)$ & $*$ & $(6.96)$ & & & & & & $(7.86)$ & $*$ & $(6.85)$ & \\
\hline \multirow{2}{*}{ RD*MI*LA } & 33.63 & & 2.68 & & & & & & 26.45 & & 0.36 & \\
\hline & $(16.35)$ & $* *$ & $(14.05)$ & & & & & & $(15.69)$ & $*$ & $(13.80)$ & \\
\hline \multirow{2}{*}{ FS } & & & & -9.85 & & -16.02 & & & -11.70 & & -14.95 & \\
\hline & & & & $(4.28)$ & $* *$ & $(3.78)$ & $* * *$ & & $(4.08)$ & $* * *$ & $(3.69)$ & $* * *$ \\
\hline \multirow{2}{*}{$\mathbf{F S}^{2}$} & & & & 97.55 & & 155.63 & & & 121.74 & & 150.79 & \\
\hline & & & & $(40.30)$ & $* *$ & $(35.69)$ & $* * *$ & & $(38.34)$ & $* * *$ & $(34.86)$ & $* * *$ \\
\hline $\mathbf{F S}^{3}$ & & & & -346.99 & & -586.27 & & & -448.34 & & -576.68 & \\
\hline
\end{tabular}




\begin{tabular}{|c|c|c|c|c|c|c|c|c|}
\hline & & $(155.73) * *$ & $(137.71)$ & $* * *$ & & $(148.12) * * *$ & $(134.62)$ & $* * *$ \\
\hline \multirow{2}{*}{$\mathbf{F S}^{4}$} & & 578.91 & 1034.66 & & & 767.38 & 1029.26 & \\
\hline & & $(286.14) * *$ & $(253.06)$ & $* * *$ & & $(272.12) * * *$ & $(247.47)$ & $* * *$ \\
\hline \multirow{2}{*}{$\mathrm{FS}^{5}$} & & -463.52 & -864.69 & & & -624.76 & -867.92 & \\
\hline & & $(249.07) *$ & $(220.59)$ & $* * *$ & & $(236.85) * * *$ & $(215.75)$ & $* * *$ \\
\hline \multirow{2}{*}{ FS $^{6}$} & & 144.06 & 276.47 & & & 196.00 & 279.58 & \\
\hline & & $(82.66) *$ & (73.35) & $* * *$ & & $(78.59) \quad * *$ & $(71.75)$ & $* * *$ \\
\hline \multirow{2}{*}{ BS } & & & & 0.04 & & 0.02 & 0.04 & \\
\hline & & & & $(0.02)$ & $* * *$ & $(0.01)$ & $(0.02)$ & $* * *$ \\
\hline \multirow{2}{*}{ BO } & & & & 0.84 & & 0.77 & 0.86 & \\
\hline & & & & $(0.19) * * *(0.17)$ & $* * *$ & $(0.18) \quad * * *$ & $(0.17)$ & $* * *$ \\
\hline \multirow[b]{2}{*}{ BP } & & & & -0.04 & & -0.04 & -0.10 & \\
\hline & & & & $(0.07)$ & & $(0.07)$ & $(0.07)$ & \\
\hline \multirow{2}{*}{ PL } & & & & -0.64 & & -0.67 & -0.82 & \\
\hline & & & & $(0.19) * * *(0.20)$ & $* * *$ & $(0.19) \quad * * *$ & $(0.20)$ & $* * *$ \\
\hline \multirow{2}{*}{ SC } & & & & 0.07 & & 0.10 & 0.05 & \\
\hline & & & & $(0.02) * * *(0.03)$ & $* *$ & $(0.02) \quad * * *$ & $(0.03)$ & $*$ \\
\hline \multirow[b]{2}{*}{ DA } & & & & -0.87 & & -1.58 & -0.97 & \\
\hline & & & & $(0.14) * * *(0.17)$ & $* * *$ & $(0.14) \quad * * *$ & $(0.17)$ & $* * *$ \\
\hline \multirow{2}{*}{ AG } & & & & -0.04 & & -0.03 & -0.03 & \\
\hline & & & & $(0.00) * * *(0.00)$ & $* * *$ & $(0.00) \quad * * *$ & $(0.00)$ & $* * *$ \\
\hline$\chi 2$ Statistic & 145.9727 & 17.3503 & & 40.9579 & & 165.1068 & & \\
\hline$\chi^{2}$ d.f. & 7 & 6 & & 7 & & 20 & & \\
\hline Prob. & 0.0000 & 0.0081 & & 0.0000 & & 0.0000 & & \\
\hline
\end{tabular}

Model 4.Upstream Industry Fixed effect of specific asset variables

Tobin's $\quad \mathrm{Q}=1.41+0.46 \mathrm{RD}+26.57 \mathrm{MI}+0.21 \mathrm{LA}+0.20 \mathrm{RD} * \mathrm{LA}-11.54 \mathrm{RD} * \mathrm{MI}-14.85 \mathrm{MI} * \mathrm{LA}+$ 33.63RD*MI*LA

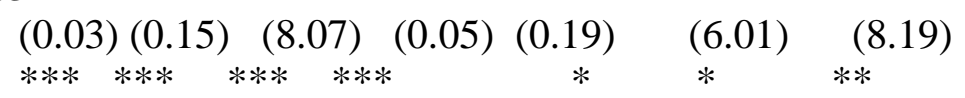

Model 5.Upstream Industry Fixed effect of internationalization degree variable Tobin's Q=1.58 -9.85FS +97.55FS2 -346.99FS3 +578.91FS4-463.52FS5 +144.06FS6

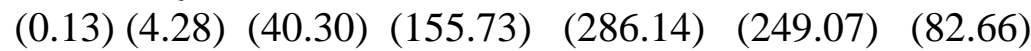
*** ** ** ** ** * * * *

Model 6.'Upstream Industry Fixed effect of other control variables

Tobin's $\mathrm{Q}=0.89+0.02 \mathrm{BS}+0.82 \mathrm{BO}-0.04 \mathrm{BP}-0.64 \mathrm{PL}+0.09 \mathrm{SC}-1.61 \mathrm{DA}-0.03 \mathrm{AG}$

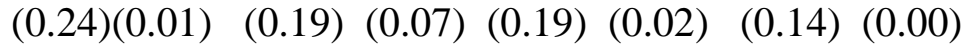

$$
\text { *** } \quad * * * \quad * * * \quad * * * \quad * * * \quad * * *
$$

Model 7.Upstream Industry the fixed effect of the whole variable

Tobin's $\quad \mathrm{Q}=0.63+0.13 \mathrm{RD}+26.99 \mathrm{MI}+0.12 \mathrm{LA}+0.36 \mathrm{RD} * \mathrm{LA}-8.42 \mathrm{RD} * \mathrm{MI}-14.52 \mathrm{MI}$ LA+ 26.45RD*MI*LA

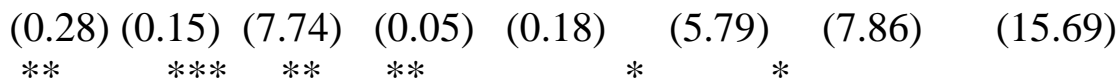

$$
\begin{aligned}
& \text {-11.70FS +121.74FS2 -448.34FS3 +767.38FS4-624.76FS5 +196.00FS6 }
\end{aligned}
$$




$$
\begin{aligned}
& \begin{array}{lllllll}
(4.08) & (38.34) & (148.12) & (272.12) & (236.85) & (78.59) \\
* * * & * * * & * * * & * * * & * * * & * * &
\end{array} \\
& +0.02 \mathrm{BS}+0.77 \mathrm{BO}-0.04 \mathrm{BP}-0.67 \mathrm{PL}+0.10 \mathrm{SC}-1.58 \mathrm{DA}-0.03 \mathrm{AG}
\end{aligned}
$$

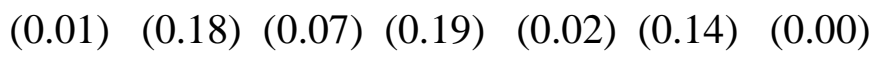

The analysis of the upstream of the electronic industry is based on the results of the analysis of each group of the models 4 to 6 , and it can be found that the effect of the interaction effect (RD * $\mathrm{MI}, \mathrm{MI} * \mathrm{LA}$ and $\mathrm{RD} * \mathrm{MI} * \mathrm{LA}$ of RD * MI, MI * LA and RD * MI * LA in both RD * MI, MI $* \mathrm{LA}$ and $\mathrm{RD} * \mathrm{MI} * \mathrm{LA}$ is not 0 ), and the degree of internationalization has been shown to be a six-power relationship. That is, the situation of the $\mathrm{W}$-type on the bottom of the board and the effect of other control variables on the performance of the company, and the structure of the board of directors has found that the scale of the board of directors and the part-time status of the board of directors are not significant, and the other variables have been the same as those of the past scholars. However, the effect of the whole on the performance of the company still needs to be obtained through the complete regression model.

\section{Specialized assets}

The upstream sample study of electronic industry found that the R \& D intensity was not significant, the marketing intensity and asset intensity showed a positive and significant relationship, while the specific assets focused on the impact of interaction, the interaction of specialized assets was similar to the results of Model 4, and the interaction results are as shown in Table 16.

\begin{tabular}{|c|c|}
\hline Specialized assets & interaction \\
\hline $\begin{array}{ll}\text { Research and } \\
\text { development } \\
\text { intensity }\end{array}$ & $0.36 \mathrm{LA}+26.45 \mathrm{MI} * \mathrm{LA}$ \\
\hline $\begin{array}{l}\text { Marketing } \\
\text { intensity } \\
\text { Capital intensity }\end{array}$ & $\begin{array}{l}26.99-14.52 \mathrm{LA}+26.45 \mathrm{RD} * \mathrm{LA} \\
0.12+0.36 \mathrm{RD}-14.52 \mathrm{MI}+26.45 \mathrm{RD} * \mathrm{MI}\end{array}$ \\
\hline
\end{tabular}

Table16. Table of interaction status of proprietary assets (Upstream Industry)

The interaction between $\mathrm{R} \& \mathrm{D}$ intensity and marketing intensity is positive and significant, which shows that when the upstream industry makes use of advertising and $\mathrm{R} \& \mathrm{D}$, it can strengthen the trust of customers and promote the growth of corporate performance, and can offset the mutually exclusive effect of expenses. However, the interaction between R \& D and assets and marketing and assets is still positive and significant.

\section{Degree of Internationalization}

In the degree of internationalization, it is found that the upstream of the electronics industry produces the $\mathrm{W}$-type at the bottom of 3 (-11.70FS 121.74FS2-448.34FS3 767.38FS4-624.76FS5 196.00FS6), and the power of $1 \geq 6$ is significant, but because the range of internationalization index is between $0 \%$ and $100 \%$, the trend diagram of its composition is $\mathrm{W}$-shaped, as shown in figure 1.

Copyright (C) 2020, Journal of Advanced Research in Economics and Administrative Sciences (JAREAS), Under a 


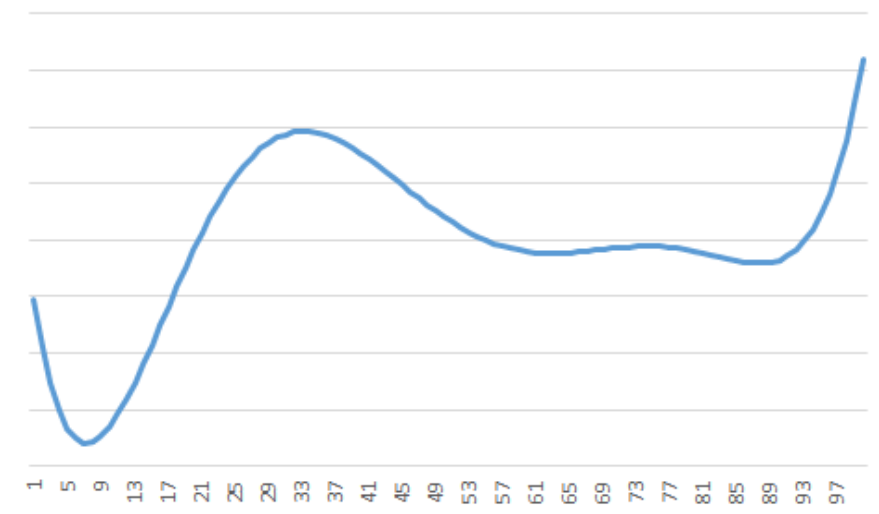

Figure 1. Chart of the impact of Upstream Industry internationalization on corporate performance.

Further analysis shows that when the enterprise increases the degree of internationalization, the impact on the performance of the company is (- 11.70 243.49FS-1345.01FS2 3069.53FS33123.82FS4 1176.00FS5), and the chart is as follows.

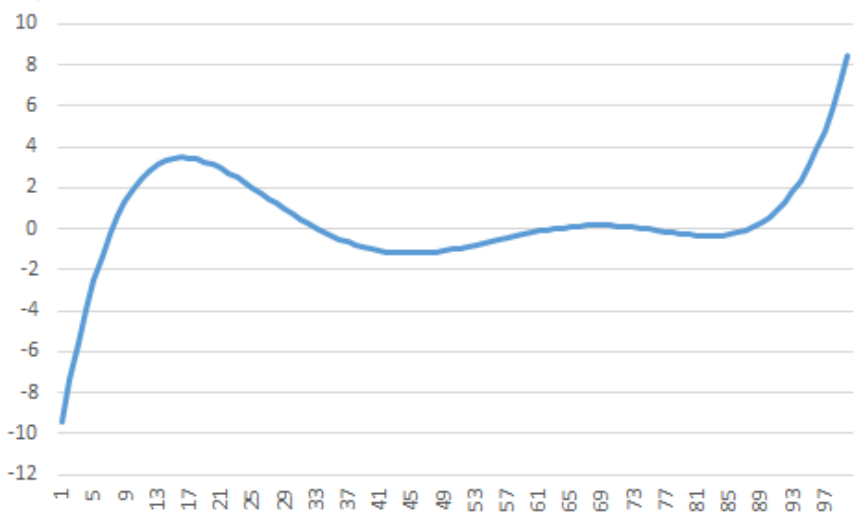

Figure 2 impact of increased internationalization of Upstream Industry on corporate performance

It can be found from figure 2 that when the internationalization is $9 \% \leq 34 \%$ and $89 \%$, the degree of internationalization can increase the performance of the company. $(65 \% \leq 75 \%$ enhancement also results in a positive increase in corporate performance, but the increase is not much.)

\section{Other control variables}

On the board structure of other control variables, this study found that the proportion of independent directors $(0.77)$ and corporate performance $(0.02)$ had a positive and significant effect on corporate performance, while the ratio of director stock pledge (0.19), debt ratio (1.08) and company age (- 0.01) showed significant negative effects, while other variables showed no significant negative effects on the performance of the company, while the proportion of independent directors (0.77) and corporate performance (0.02) showed significant negative effects on the performance of the board of directors (0.19), debt ratio (-1.08) and age of the company (-0.01).

The analysis results of Midstream Industry can be seen from Table 17. The four groups of factors for analyzing the degree of internationalization in China are suitable for random effects, while the others are suitable for analysis with fixed effect models, such as model $8 \leq 11$. 
Table17. Midstream Industry Fixed and Random Effects Model

\begin{tabular}{|c|c|c|c|c|c|c|c|c|c|c|c|c|c|}
\hline \multirow[t]{3}{*}{ Variable } & \multicolumn{2}{|l|}{ FIXED } & \multicolumn{2}{|c|}{ RANDOM } & \multicolumn{2}{|l|}{ FIXED } & RANDOM & \multirow{2}{*}{$\frac{\text { FIXED }}{1.36}$} & RANDOM & \multicolumn{2}{|l|}{ FIXED } & \multicolumn{2}{|c|}{ RANDOM } \\
\hline & 0.98 & & 1.07 & & 1.14 & & 1.23 & & 2.14 & 1.35 & & 2.25 & \\
\hline & $(0.01)$ & $* * *$ & $*(0.02)$ & $* * *$ & $(0.05)$ & $* * *$ & $(0.05) *$ & $* * *(0.12) * * *$ & $(0.18)$ & $* * *(0.13)$ & $* * *$ & $(0.18)$ & $* * *$ \\
\hline \multirow[t]{2}{*}{ RD } & 1.31 & & -0.29 & & & & & & & 0.85 & & -0.59 & \\
\hline & $(0.18)$ & $* * *$ & $*(0.19)$ & & & & & & & $(0.18)$ & $* * *$ & $(0.19)$ & $* * *$ \\
\hline \multirow[t]{2}{*}{ MI } & 7.73 & & 1.32 & & & & & & & 5.70 & & -2.82 & \\
\hline & (2.39) & $* * *$ & $*(2.70)$ & & & & & & & $(2.37)$ & $* *$ & $(2.68)$ & \\
\hline \multirow[t]{2}{*}{ LA } & 0.31 & & 0.24 & & & & & & & 0.29 & & 0.21 & \\
\hline & $(0.02)$ & & $*(0.02)$ & $* * *$ & & & & & & $(0.02)$ & $* * *$ & $(0.02)$ & $* * *$ \\
\hline \multirow[t]{2}{*}{ RD*LA } & -0.56 & & -0.02 & & & & & & & -0.47 & & 0.05 & \\
\hline & $(0.10)$ & $* * *$ & $*(0.09)$ & & & & & & & $(0.10)$ & $* * *$ & (0.09) & \\
\hline \multirow[t]{2}{*}{ RD*MI } & -20.12 & & -8.87 & & & & & & & -12.63 & & -2.22 & \\
\hline & (10.68) & $*$ & $(10.07)$ & & & & & & & (10.48) & & (9.88) & \\
\hline \multirow[t]{2}{*}{ MI*LA } & -8.63 & & -8.80 & & & & & & & -11.57 & & -9.91 & \\
\hline & (3.89) & $* *$ & $(3.52)$ & $* *$ & & & & & & $(3.81)$ & $* * *$ & $(3.46)$ & $* * *$ \\
\hline \multirow{2}{*}{ RD*MI*LA } & 7.82 & & 7.12 & & & & & & & 6.93 & & 5.57 & \\
\hline & $(5.58)$ & & $(5.07)$ & & & & & & & $(5.47)$ & & (4.97) & \\
\hline \multirow{2}{*}{ FS } & & & & & -3.38 & & -2.43 & & & -4.00 & & -3.00 & \\
\hline & & & & & $(1.21)$ & $* * *$ & $(1.16) *$ & $* *$ & & (1.17) & $* * *$ & (1.12) & $* * *$ \\
\hline \multirow{2}{*}{ FS2 } & & & & & 22.89 & & 15.65 & & & 25.97 & & 17.92 & \\
\hline & & & & & (7.89) & $* * *$ & $(7.53) *$ & $* *$ & & (7.61) & $* * *$ & $(7.25)$ & $* *$ \\
\hline \multirow{2}{*}{ FS3 } & & & & & -56.10 & & -38.85 & & & -62.55 & & -41.92 & \\
\hline & & & & & (19.70) & $* * *$ & $(18.79) *$ & & & (18.97) & $* * *$ & (18.09) & $* *$ \\
\hline \multirow{2}{*}{ FS4 } & & & & & 58.16 & & 40.91 & & & 64.19 & & 42.47 & \\
\hline & & & & & (20.99) & $* * *$ & $(20.09) *$ & & & (20.21) & $* * *$ & (19.33) & $* *$ \\
\hline \multirow{2}{*}{ FS5 } & & & & & -21.69 & & -15.53 & & & -23.73 & & -15.62 & \\
\hline & & & & & (8.04) & $* * *$ & $(7.73) *$ & $* *$ & & (7.74) & $* * *$ & (7.44) & $* *$ \\
\hline \multirow{2}{*}{ BS } & & & & & & & & 0.02 & 0.04 & 0.02 & & 0.04 & \\
\hline & & & & & & & & $(0.01) * * *$ & $(0.01)$ & $* * *(0.01)$ & $* * *$ & $(0.01)$ & $* * *$ \\
\hline \multirow{2}{*}{ BO } & & & & & & & & 0.36 & 0.41 & 0.34 & & 0.44 & \\
\hline & & & & & & & & $(0.08) * * *$ & $(0.08)$ & $* * *(0.08)$ & $* * *$ & $(0.08)$ & $* * *$ \\
\hline & & & & & & & & -0.05 & -0.01 & -0.05 & & 0.00 & \\
\hline Dr & & & & & & & & $(0.03)$ & $(0.04)$ & $(0.03)$ & & $(0.04)$ & \\
\hline D & & & & & & & & -0.02 & -0.13 & -0.02 & & -0.11 & \\
\hline $1 \mathrm{H}$ & & & & & & & & $(0.07)$ & $(0.08)$ & $(0.07)$ & & $(0.07)$ & \\
\hline & & & & & & & & 0.00 & -0.07 & -0.01 & & -0.07 & \\
\hline $\mathbf{S C}$ & & & & & & & & $(0.01)$ & $(0.01)$ & $* * *(0.01)$ & & $(0.01)$ & $* * *$ \\
\hline & & & & & & & & -0.91 & -0.60 & -0.86 & & -0.63 & \\
\hline DA & & & & & & & & $(0.06) * * *$ & $(0.08)$ & $* * *(0.06)$ & $* * *$ & $(0.07)$ & $* * *$ \\
\hline
\end{tabular}




\begin{tabular}{|c|c|c|c|c|c|c|c|c|}
\hline \multirow{2}{*}{ AG } & & & -0.01 & -0.01 & 0.00 & & -0.01 & \\
\hline & & & \multicolumn{2}{|c|}{$(0.00) * * *(0.00)$} & $* * *(0.00)$ & $* * *$ & $(0.00)$ & $* * *$ \\
\hline$\chi^{2}$ Statistic & 108.2351 & 9.0560 & 63.97 & & 172.07 & & & \\
\hline$\chi^{2}$ d.f. & 7.0000 & 5.0000 & 7.000 & & 19.000 & & & \\
\hline Prob. & 0.0000 & 0.1069 & 0.000 & & 0.0000 & & & \\
\hline
\end{tabular}

Model 8.Midstream Industry The fixed effect of the specific asset variable

Tobin's $\quad \mathrm{Q}=0.98+1.31 \mathrm{RD}+7.73 \mathrm{MI}+0.31 \mathrm{LA}-0.56 \mathrm{RD} * \mathrm{LA}-20.12 \mathrm{RD} * \mathrm{MI}-8.63 \mathrm{MI} * \mathrm{LA}+$ 7.82RD*MI*LA

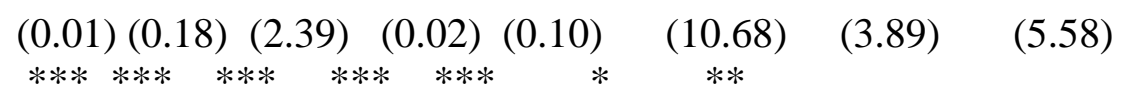

Model 9.Midstream Industry Fixed effect of internationalization degree variable

Tobin's $\mathrm{Q}=1.23-2.43 \mathrm{FS}+15.65 \mathrm{FS} 2$-38.85FS3 +40.91FS4-15.53FS5

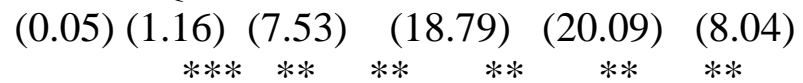

Model 10.Midstream Industry Fixed effect of other control variables

Tobin's $\mathrm{Q}=1.36+0.02 \mathrm{BS}+0.36 \mathrm{BO}-0.05 \mathrm{BP}-0.02 \mathrm{PL}+0.00 \mathrm{SC}-0.91 \mathrm{DA}-0.01 \mathrm{AG}$

$$
\begin{aligned}
& \begin{array}{lllllll}
(0.12) & (0.01) & (0.08) & (0.03) & (0.07) & (0.01) & (0.06)
\end{array} \\
& * * * * * * \quad * * * \quad * * * * * *
\end{aligned}
$$

Model 11.Midstream Industry Fixed effect of full variable

Tobin's $\quad \mathrm{Q}=1.35+0.85 \mathrm{RD}+5.70 \mathrm{MI}+0.29 \mathrm{LA}-0.47 \mathrm{RD} * \mathrm{LA}-12.63 \mathrm{RD} * \mathrm{MI}-11.57 \mathrm{MI} * \mathrm{LA}+$ 6.93RD*MI*LA

$$
\begin{aligned}
& \begin{array}{llll}
(0.13)(0.18) & (2.37)(0.02) & (0.10) \\
* * * * * * & * * * * * * * * * & (10.48)
\end{array} \\
& \text {-4.00FS +25.97FS2 -62.55FS3 +64.19FS4-23.73FS5 }
\end{aligned}
$$

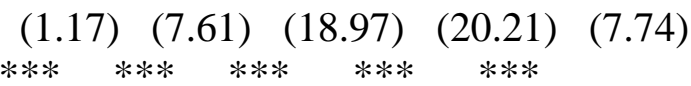

$$
\begin{aligned}
& +0.02 \mathrm{BS}+0.34 \mathrm{BO}-0.05 \mathrm{BP}-0.02 \mathrm{PL}+0.01 \mathrm{SC}-0.86 \mathrm{DA}-0.00 \mathrm{AG}
\end{aligned}
$$

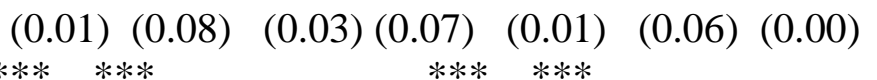

According to the results of model 8 / 11, we can find that the R \& D intensity of specialized asset variable (1.31), marketing intensity (7.73) and capital intensity (0.31) are positive and significant, and the interaction effect of cross-specific asset variables (RD*LA, RD*MI and MI*LA is significantly not 0 ), but the interaction of three variables is not significant. The degree of internationalization showed a nonlinear five-power significant situation, while other control variables found that the part-time status of board supervisors was not significant in the structure of the board of directors, and the other two managers were found to have no significant part-time status in the board structure of the board of directors. The size of the board of directors (0.02) and the proportion of independent supervisors $(0.36)$ were significantly positive, while other variables found that the size of the company was not significant, and the debt ratio (- 0.86) and the age of the company (- 0.00) showed significant negative effects. However, the overall impact on corporate performance still needs a complete regression model to get the most real answer.

The results of model 11 are as follows:

Specialized assets

Copyright (C) 2020, Journal of Advanced Research in Economics and Administrative Sciences (JAREAS), Under a 
In this study, the effect of interaction on specific assets is studied, and the results of the interaction are shown in Table 18.

Table18.Table of interaction status of Midstream Industry specific assets

\begin{tabular}{l|l}
\hline Specialized assets & interaction \\
\hline $\begin{array}{l}\text { Research and } \\
\text { development } \\
\text { intensity }\end{array}$ & $0.85-0.47 \mathrm{LA}$ \\
$\begin{array}{l}\text { Marketing } \\
\text { intensity } \\
\text { Capital intensity }\end{array}$ & $0.29-0.47 \mathrm{RD}-11.57 \mathrm{MI}$ \\
\hline
\end{tabular}

The analysis results show that all of the three attributes have a positive and significant impact on corporate performance, but in the interaction, it is found that the capital intensity has a negative effect with the other two attributes. And the interaction between the three attributes did not occur significantly.

Degree of internationalization

In the degree of internationalization, it is found that the fifth power is significant in the whole period (- 4.00FS 25.97FS2-62.55FS3 64.19FS4-23.73FS5), but since the scope of internationalization index is between $0 \%$ and $100 \%$, the composition of the index is shown in figure 3 .

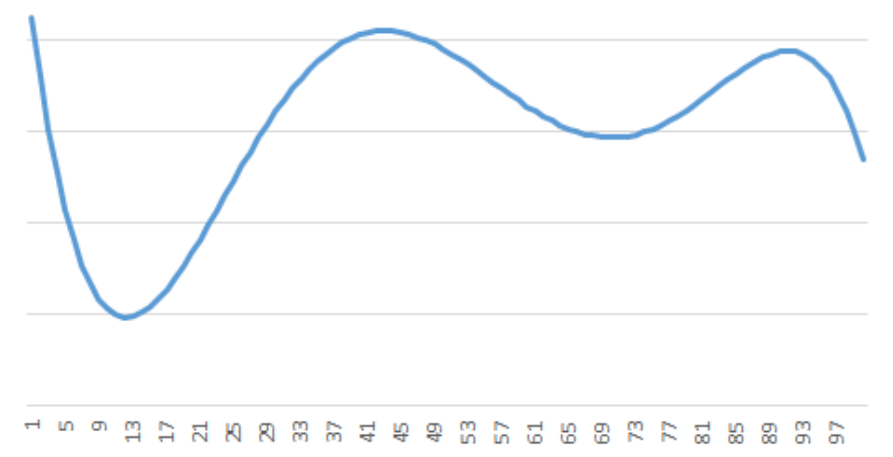

Figure 3.The impact of Midstream Industry's internationalization on the company's performance.

Further analysis shows that the impact on corporate performance is-4.00 51.95FS1-187.64FS2 256.74FS3 / 118.64FS4 when the enterprise increases the degree of internationalization, and the trend chart is shown in figure 4 below.

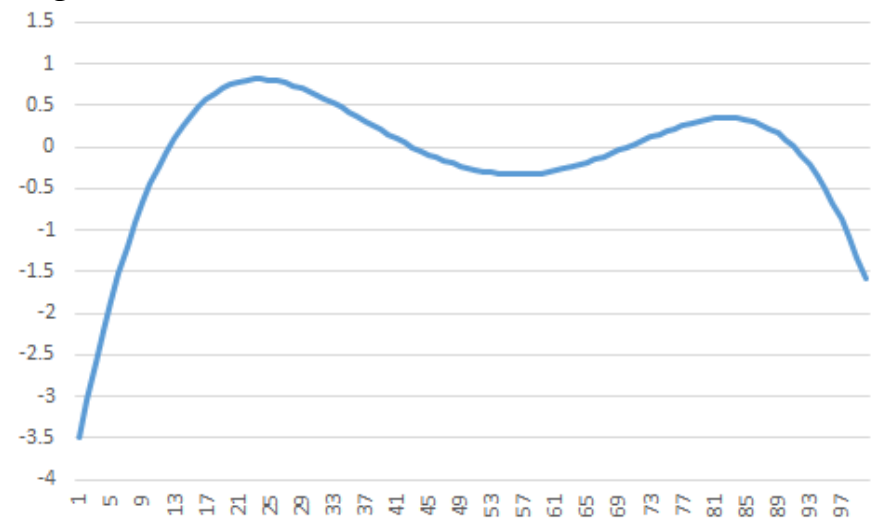

Figure 4 impact of increased internationalization of Midstream Industry on corporate performance

Copyright (C) 2020, Journal of Advanced Research in Economics and Administrative Sciences (JAREAS), Under a 
It can be found from figure 4 that when the internationalization is $13 \% \leq 43 \%$ and $71 \% \leq 91 \%$, the performance of the company can be increased only by strengthening the degree of internationalization. In the structure of the board of directors, this study found that there was no significant effect on the performance of the company and (0.72) showed a significant positive effect on the performance of the company, but the part-time situation of the supervisor $(-0.16)$ had a negative and significant effect on the performance of the company.

\section{Other control variables}

The size of board of directors (0.02) and the proportion of independent directors $(0.34)$ on other control variables have positive significance on corporate performance, while debt ratio (- 0.86) and company age (- 0.00) have significant negative effects, while other variables have no significant effect.

The downstream analysis results of the electronic industry can be seen from Table 19. The four groups of analysis are suitable for the analysis of fixed effect models, such as model $12 \leq 15$.

Table 19.Downstream Industry Fixed and Random Effects Model

\begin{tabular}{|c|c|c|c|c|c|c|c|c|}
\hline Variable & FIXED & RANDOM & FIXED & RANDOM & FIXED & RANDOM & FIXED & RANDOM \\
\hline \multirow{2}{*}{$\mathbf{C}$} & 1.06 & 1.15 & 1.04 & 1.19 & 1.04 & 1.56 & 0.95 & 1.64 \\
\hline & $(0.01)$ & $* * *(0.03) * * *$ & $(0.03) * * *$ & $(0.04) * * *$ & $(0.11)$ & $* * *(0.17) * * *$ & $(0.12) * * *$ & $(0.18) * * *$ \\
\hline \multirow{2}{*}{ RD } & 0.33 & -0.23 & & & & & -0.04 & -0.34 \\
\hline & $(0.10)$ & $* * *(0.10) * *$ & & & & & $(0.10)$ & $(0.10) * * *$ \\
\hline \multirow{2}{*}{ MI } & 8.25 & -3.99 & & & & & 3.93 & -5.93 \\
\hline & $(1.51)$ & $* * *(1.62) * *$ & & & & & $(1.47) * * *$ & $(1.60) * * *$ \\
\hline \multirow{2}{*}{ LA } & 0.11 & 0.06 & & & & & 0.08 & 0.04 \\
\hline & $(0.02)$ & $* * *(0.02) * * *$ & & & & & $(0.02) * * *$ & $(0.02) * *$ \\
\hline \multirow{2}{*}{ RD*LA } & -0.15 & 0.05 & & & & & -0.09 & 0.07 \\
\hline & $(0.12)$ & $(0.10)$ & & & & & $(0.12)$ & $(0.10)$ \\
\hline \multirow{2}{*}{ RD*MI } & -3.50 & 4.74 & & & & & 1.32 & 6.98 \\
\hline & (4.07) & (3.62) & & & & & $(3.90)$ & $(3.54) * *$ \\
\hline \multirow{2}{*}{ MI*LA } & -2.15 & 0.90 & & & & & -2.03 & 1.62 \\
\hline & (1.88) & (1.59) & & & & & (1.79) & (1.56) \\
\hline \multirow{2}{*}{ RD*MI*LA } & -0.21 & -2.67 & & & & & -0.47 & -3.63 \\
\hline & (3.93) & $(3.32)$ & & & & & $(3.75)$ & $(3.25)$ \\
\hline \multirow{2}{*}{ FS } & & & 1.43 & 0.56 & & & -0.01 & -0.01 \\
\hline & & & $(0.59) * *$ & $(0.58)$ & & & $(0.00) * * *$ & $(0.00) * * *$ \\
\hline \multirow{2}{*}{ FS2 } & & & -6.70 & -3.64 & & & 1.33 & 0.42 \\
\hline & & & $(2.70) * *$ & $(2.60)$ & & & $(0.57) * *$ & $(0.57)$ \\
\hline \multirow{2}{*}{ FS3 } & & & 10.63 & 5.99 & & & -5.75 & -2.71 \\
\hline & & & $(4.10) * * *$ & $(3.94)$ & & & $(2.57) * *$ & $(2.53)$ \\
\hline \multirow{2}{*}{ FS4 } & & & -5.31 & -3.04 & & & 8.75 & 4.38 \\
\hline & & & $(2.00) * * *$ & $(1.92)$ & & & $(3.92) * *$ & $(3.84)$ \\
\hline BS & & & & & -0.01 & 0.02 & -4.29 & -2.17 \\
\hline
\end{tabular}




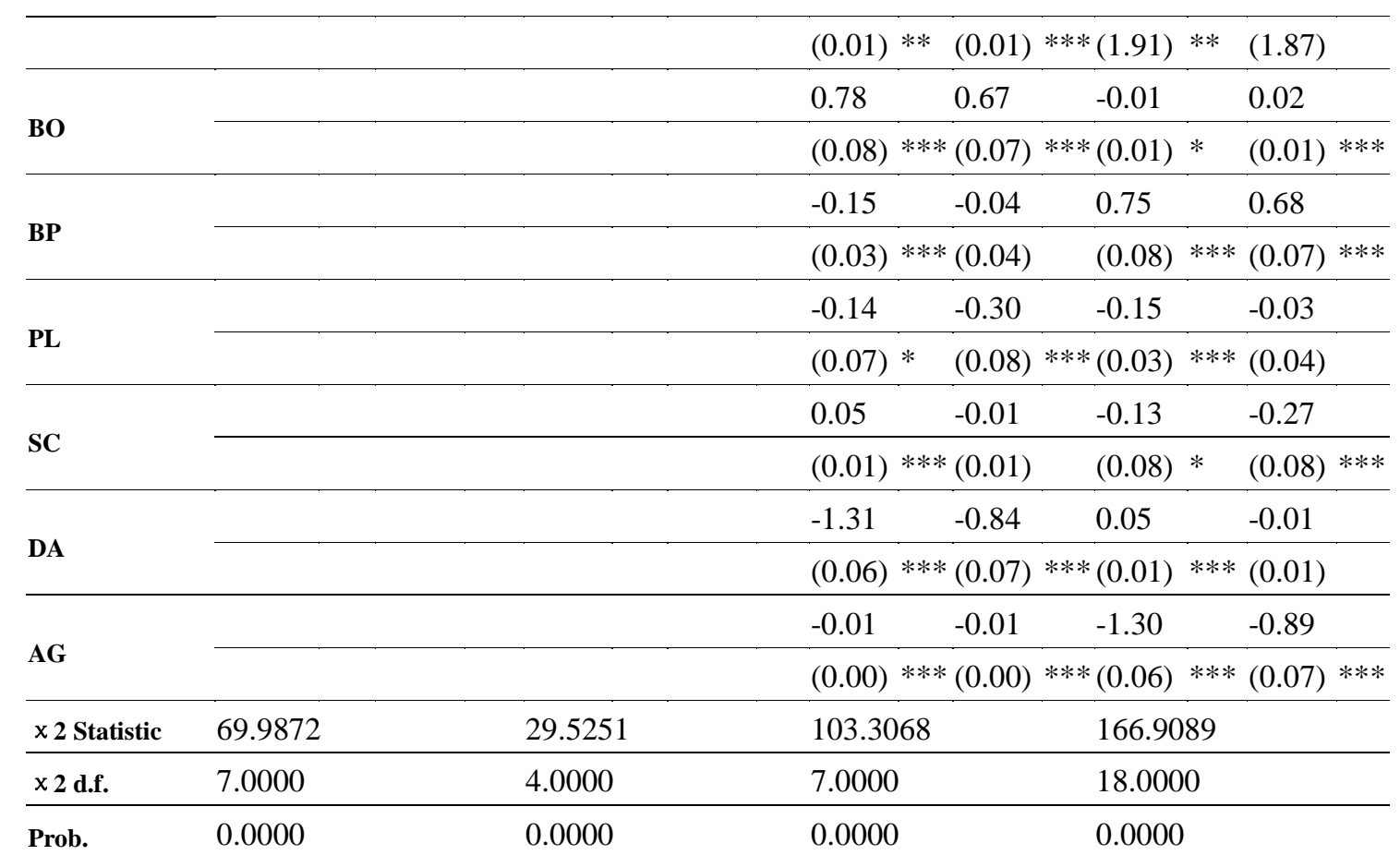

Model 12.Fixed effect of Downstream Industry specific Asset variable

Tobin's $\quad \mathrm{Q}=1.06+0.33 \mathrm{RD}+8.25 \mathrm{MI}+0.11 \mathrm{LA}-0.15 \mathrm{RD} * \mathrm{LA}-3.50 \mathrm{RD} * \mathrm{MI}-2.15 \mathrm{MI} * \mathrm{LA}-0.21$ $\mathrm{RD} * \mathrm{MI} * \mathrm{LA}$
$(0.01)(0.10) \quad(1.51)(0.02)$
$* * * * * * * * * * * *$
$(0.12) \quad(4.07)$
$(1.88)$

Model 13.Random effect of Downstream Industry internationalization degree variable

Tobin's $\mathrm{Q}=1.04+1.43 \mathrm{FS}-6.70 \mathrm{FS} 2+10.63 \mathrm{FS} 3-5.31 \mathrm{FS} 4$

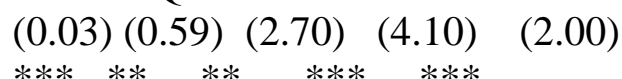

Model 14.Fixed effect of Downstream Industry other control variables

Tobin's $\mathrm{Q}=1.04-0.01 \mathrm{BS}+0.78 \mathrm{BO}-0.15 \mathrm{BP}-0.14 \mathrm{PL}+0.05 \mathrm{SC}-1.31 \mathrm{DA}-0.01 \mathrm{AG}$

(0.11) (0.01) (0.08) (0.03) (0.07) (0.01) (0.06) $(0.00)$

*** $* * \quad * * * \quad * * * \quad * \quad * * * \quad * * * \quad * * *$

Model 15.The Fixed Effect of the Full-variable of the Downstream

Tobin's $\quad \mathrm{Q}=0.95 \quad-0.04 \mathrm{RD}+3.93 \mathrm{MI}+0.08 \mathrm{LA}-0.09 \mathrm{RD} * \mathrm{LA}+1.32 \mathrm{RD} * \mathrm{MI}-2.03 \mathrm{MI} * \mathrm{LA} \quad$ $0.47 \mathrm{RD} * \mathrm{MI} * \mathrm{LA}$

$$
\begin{aligned}
& (0.12)(0.10) \text { (1.47) (0.02) (0.12) (3.90) (1.79) (3.75) } \\
& +1.33 \mathrm{FS}-5.75 \mathrm{FS} 2+8.75 \mathrm{FS} 3-4.29 \mathrm{FS} 4 \\
& \begin{array}{ccc}
(0.57) & (2.57)(3.92) & (1.91) \\
* * & * * \quad * * \quad * *
\end{array} \\
& -0.01 \mathrm{BS}+0.75 \mathrm{BO}-0.15 \mathrm{BP}-0.13 \mathrm{PL}+0.05 \mathrm{SC}-1.30 \mathrm{DA}-0.01 \mathrm{AG} \\
& \text { (0.01) (0.08) (0.03) (0.08) (0.01) (0.06) (0.00) }
\end{aligned}
$$

The analysis of the downstream of the electronic industry shows that the variables of specialized assets have a positive effect on the performance of the company (but the R \& D intensity is not significant), but the effect of the interaction is not significant, and the degree of internationalization shows the condition of $\mathrm{W}$ type, the results of the downstream analysis of the electronic industry show that the variables of specialized assets have a positive effect on the 
performance of the company (but the intensity of $\mathrm{R} \& \mathrm{D}$ is not significant). The influence of other control variables on corporate performance is about the same as that of previous scholars (only the board size is negative and significant). However, the overall impact on corporate performance still needs a complete regression model (model 15) to get the most real answer.

The results of model 15 are as follows:

\section{Specificity Assets}

Only the marketing intensity (3.93) and the capital strength (0.08) of the specific assets have a significant positive effect. And the R \& D strength is not significant. And the interaction is not significant.

\section{Degree of internationalization}

In the international level, it was found that there was a W-type in the full-time period (1.33 FS5.75 FS2 + 8.75 FS3-4.29 FS4), and the 1-to-4 th power was significant, but since the range of the internationalization index was between $0 \%$ and $100 \%$, the trend chart constituted by it presented the W-type, as shown in Figure 5.

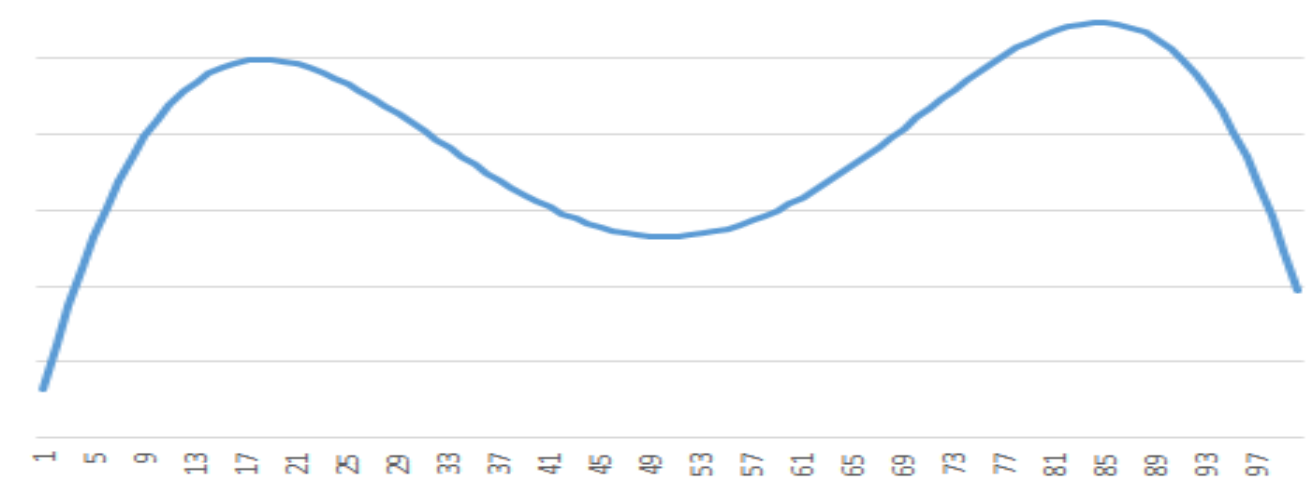

Figure 5. the impact of Downstream Industry internationalization on corporate performance.

Further analysis shows that the impact on corporate performance is $1.33-11.51 \mathrm{FS} 1$ $+26.25 \mathrm{FS} 2-17.16 \mathrm{FS} 3$ when the enterprise increases the degree of internationalization, and the chart is shown in figure 6 below.

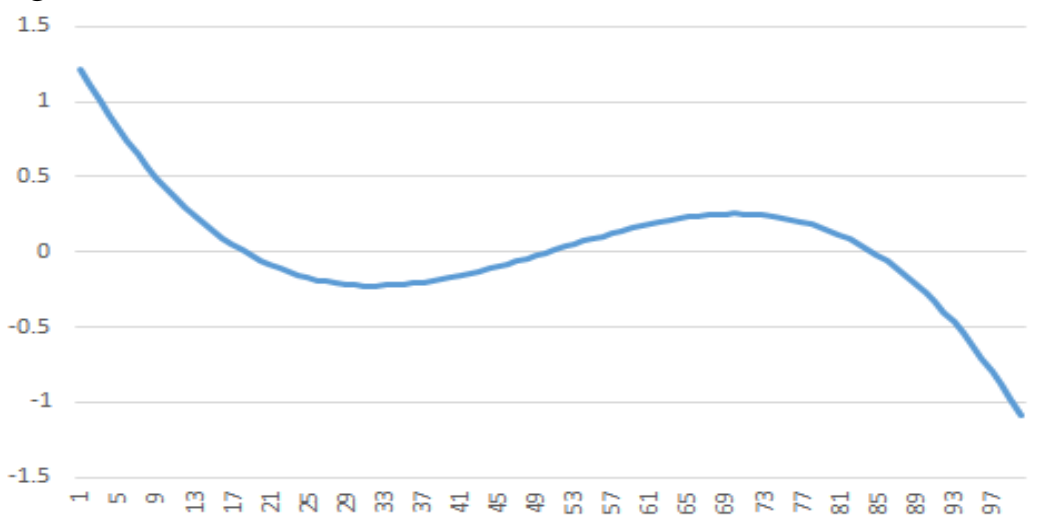

Figure 6 impact of increased internationalization of Downstream Industry on corporate performance 
It can be found from FIG.6 that when the enterprise is engaged in the enhancement of the internationalization between $0 \% \sim 18 \%$ and $51 \% \sim 84 \%$, the degree of internationalization will be strengthened to increase the company's performance.

\section{Other control variables}

In other control variables, the proportion of independent directors (0.75) and the size of the company (- 0.03) showed positive significance with the performance of the company. The size of the board of directors $(-0.01)$, the part-time status of the board of directors $(-0.15)$, the ratio of board of directors' stock pledge (- 0.13), the proportion of liabilities $(-1.30)$ and the age of the company (-0.01) showed negative significance with the company performance.

\section{Conclusion and Suggestion}

The purpose of this study is to explore the interaction of corporate proprietary attributes in Taiwan's electronics industry chain and the impact of internationalization on corporate performance. The electronic industrial chain is divided into upstream, CNOOC and downstream, upstream industry is semiconductor industry, middle reaches is electronic component level optoelectronics industry, computer and periphery, communication network road industry, electronic communication industry, information service industry and other electronic industries, the research period is 15098 in the 20 years from 1998 to 2017.

This study found that the R \& D intensity has a significant impact only in the upstream industry of the electronics industry, and there is no significant occurrence in the middle and lower reaches of the electronics industry, which is a very strange situation in Taiwan's electronics industry. However, because many scholars studied the R \& D intensity has a deferred effect in the past, it may have a deferred effect in the middle and lower reaches of the R \& D intensity, so that the R $\&$ D intensity does not have a significant effect in the "first year". However, the interaction of company proprietary attributes did not occur downstream, and the two interactions upstream showed negative results, which showed that the company cost was limited and produced mutually exclusive effect, but in the middle reaches, the asset intensity increased. It can make the R \& D intensity have a bonus effect on the performance of the company. And the interaction of the three attributes of the midstream electronics industry can also produce multiplication effect.

The second is the international situation, the research internationalization of the past scholars has almost positive, negative, positive $\mathrm{U}$, reverse $\mathrm{U}$ or $\mathrm{W}$ type. The research has found that the degree of internationalization has a higher effect on the performance of the company, but no matter how high it is connected, It seems that the internationalization in the stage of $10 \% \sim 50 \%$ and more than $80 \%$ increase the degree of internationalization can increase the company's performance.

In other variables, this study also found that no matter how the industry is classified, the proportion of debt and the age of the company have a negative impact, especially the age of the company, which seems to be different from that of other scholars. If we cooperate with the size of the company (some of which are not significant), it should be possible to use Taiwan, most of which belong to small and medium-sized enterprises, and small and medium-sized enterprises are more flexible, which results in a negative and significant situation of the age of the company. The fact that the size of the company is not significant can be explained.

Copyright (C) 2020, Journal of Advanced Research in Economics and Administrative Sciences (JAREAS), Under a 


\section{Conflict of Interests}

The authors declare no conflict of interest.

\section{Funding}

For this research work, the authors did not receive funds from any organization.

\section{References}

Agrawal, A. and Knoeber C. R. , (1996), "Firm Performance and Mechanisms to Control Agency Problems between Managers and Shareholders," Journal of Financial and Quantitative Analysis, 3, 377-397.

Bae, S.C., Park, Bell J.C., Xiaohong and Wang (2008) "Multinationali ty, R\&D intensity and firm performance: evidence from U.S. manufacturing firms". Multinational Business Review 16(1): 53-77

Barney, J. (1991), "Firm resources and sustained competitive advantage", Journal of Management, 17(1), pp. 99-120.

Baysinger and Hoskisson, (1990), "The composition of boards of direc tors and strategic control: effects on corporate strategy," Academy of Management Review, 15 (1), 72-87.

Bradley, M., Jarrell, G., Kim, E., H., (1984), "On the existence of an optimal capital structure: theory and evidence", Journal of Finance, 39, 857-878.

Brouthers, L. E., Nakos, G. Hadjimarcou, J. and Brouthers, K. D. (2009), "Key factors for successful export performance for small firms", Journal of International Marketing, 17(3), pp. 21-38.

Caves, R. E. (1971), "International corporations: The industrial eco nomics of foreign investment", Economica, 38, pp. 1-27.

Caves, R. E. (1996), Multinational Enterprise And Economic Analysis, XCambridge, MA: Harvard University Press.

Chan, S.-H., J. D. Martin, and J. W. Kensinger (1990), "Corporate re search and development expenditures and share values," Journal of Financial Economics, 26(2), pp.255-276.

Chari, M.D.R., Devaraj, S. and David, P. (2007) "International diversi fication and firm performance: Role of information technology investments", Journal of World Business 42(2): 184-197.

Claessens, S., Djankov, S., \& Lang, L. H. P. (2000), “The separation of ownership and control in East Asian corporations." Journal of Financial Economics, 58(1-2), 81-112

Core., J. E., R. W. Holthausen, and D. F. Larcker.. Corporate Govern ance, (1999), "Chief Executive Officer Compensation, and Firm Performance," Journal of Financial Economics, 51, 371-406.

Crutchley, C. E. and R. S. Hansen, (1989), "A Test of the Agency Theo ry of Managerial Ownership, Corporate Leverage and Corporate Dividends," Financial Management, 18, 36-46.

D., Josheski, and M., M., Sopova,(2013), Market Value of the Firms and R\&D Investment: Theoretical Overview and Empirical Estimation for the Panel of Countries, Intrernational Journal of Business Management and Administration,2(3), 55-63.

Delios, A. and Beamish, P.W. (1999) "Georgaphic scope, product diver sification, and the corporate performance of Japanese firms ", Strategic Management Journal 20(8),711727.

Dess, Gregory G., Anil Gupta, Jean Francois Hennart, and Charles W. L. Hill (1995), "Conducting and Integrating Strategy Research at the International, Corporate, and Business Levels: Issues and Directions," Journal of Management, 21 (3), 357-393.

Fama, (1980), "Agency problems and the theory of the firm," Journal of Political Economy, 88

Copyright (C) 2020, Journal of Advanced Research in Economics and Administrative Sciences (JAREAS), Under a 
(2), 88-307.

Ferris, Jagannathan, and Pritchard, (2003), "Too busy to mind the busi ness? Monitoring by directors with multiple board appointments," Journal of Finance, 58, 1087-1111.

Fich and Shivdasani, (2005), "Are Busy Boards Effective Monitors," Journal of Finance, 61 (2), 689-724.

Filatotchev, I. and Piesse, J., (2009), "R\&D, internationalization and growth of newly listed firms: European evidence", Journal of International Business Studies 40(8): 1260-1276.

Franzen, L. and Radhakrishnan, S., (2009), The value relevance of R\&D across profit and loss firms, Journal of Accounting and Public Policy, 28, 16-32.

Gaur A, and Kumar V (2009) International Diversification, Business Group Affiliation and Firm Performance: Empirical Evidence from India. British Journal of Management, 20 (2), 172-186.

Grubaugh, S. G. (1987), "Determinants of direct foreign investment", The Review of Economics and Statistics, 69(1), 149-152.

Hausman, Jerry A. and William E. Taylor, 1981, "Panel Data and Un observable Individual Effects," Econometrica, 49, No. 6,1377-1398

Hennart, J.-F. (1991), The Transaction Cost Theory of The Multinational Enterprise. in The Nature of the Transnational Firm, C. Pitelis and R. Sugden (Eds.), London: Routledge,.81-116.

Hermalin, B. E. and M. Weisbach, (1991), "The Effects of Board Com position and Direct Incentives on Firm Performance," Financial Management , 20, 101-112.

Hirschey, Mark, Connolly, Robert A, (2005), "Firm Size and the Effect of R\&D on Tobin's Q", $R \&$ D Management, 35(2), 217-223.

Hirschey, M., and Weygandt, J., (1993), Amortization Policy for Adver tising and R\&D Expenditures, Journal of Accounting Research, 326-335.

Hitt, M. A., R. E. Hoskisson, and R. D. Ireland (1994), “A mid-range theory of the interactive effects of international and product diversification on innovation and performance," Journal of Management, 20(2), pp.297-326.

Hitt, M.A., Hoskisson, R.E., and Kim, H. (1997) "International diversi fication: Effects on innovation and firm performance in product-diversified firms", Academy of Management Journal 40(4): 767-798.

Hsu, C. C. and Pereira, A. (2008), "Internationalization and perfor mance: The moderating effects of organizational learning", Omega-International Journal of Management Science, 36(2), pp. 188-205.

Jensen, M.C. (1986) Agency Costs of Free Cash Flow, Corporate Fi nance and Takeover. American Economic Review, 76, 323-329.

Jensen, G. R., D. P. Solberg, and T. S. Zorn, (1992), "Simultaneous De termination of Insider Ownership, Debt, and Dividend Policies," Journal of Financial and Quantitative Analysis, 27, 247-263.

Jung, Y. (1991) "Multinationality and profitability", Journal of Business Research 23 (2):17987.

Kafouros, M.I., Buckley, P.J., Sharp, J.A. and Wang, C.Q. (2008) "The role of internationalization in explaining innovation performance", Technovation 28(1-2) : 6374.

Klette, T., (1996), R\&D, Scope Economices, and Plant Performance, R\&D journal of Economics, 27(3), 502-522.

Kotabe, M., Srinivasan, S.S. and Aulakh, P.S. (2002) "Multinationality and firm performance: The moderating role of R\&D and marketing capabilities", Journal of International Business Studies 33(1), 79-97.

Copyright (C) 2020, Journal of Advanced Research in Economics and Administrative Sciences (JAREAS), Under a Creative Commons Attribution 4.0 International License 
Kravis, I. B. and R. E. Lipsey (1992), "Sources of competitiveness of the United States and of its multinational firms," The Review of Economics and Statistics, 74(2), 193-201.

La Porta, R., F. Lopez-de-Silanes, A. Shleifer, and R. W. Vishny, (2002), "Investor Protection and Corporate Valuation," Journal of Finance, 57, 1147-1171.

Lall, S. (1980), "Monopolistic Advantages and Foreign Involvement by U.S. Manufacturing Industry," Oxford Economic Papers, 32(1), 102-122.

Lev, B., and Aboody, D., (2001), R\&D productivity in the chemical industry, Working paper.

Li, L. (2005), "Is regional strategy more effective than global strategy in the US service industries? ", Management International Review, 45(1), 37.

Lu, J.W. and Beamish, P.W. (2004) "International diversification and firm performance: The SCURVE hypothesis", Academy of Management Journal, 47(4), 598-609.

Mank, D. A. and H. E. Nystrom (2001), "Decreasing returns to share holders from R\&D spending in the computer industry," Engineering Management Journal, 13(3), 3-8.

McConnell. H.Servaes (1990),"Additional Evdence on Equity Owner ship and Corporate Value," Journal of Financial Economicss, 25, 595-612

Missaka Warusawitharana, (2015), Research and development, profits and firm value: A structural estimation", Quantitative Economics, 6(2), 531-565.

Morck, R. and Yeung, B. (1991) "Why investors value multinationality ", Journal of Business 64(2), 165-187.

Morck, R., A. Shleifer, and R.W. Vishny,, (1988), "Management Own ership and Market Valuation: An Empirical Analysis," Journal of Financial Economics, 20, 293-315.

Morck, R., A. Shleifer, and R.W. Vishny,, (1988), "Management Own ership and Market Valuation: An Empirical Analysis," Journal of Financial Economics, 20, 293-315.

Mundlak, A.(1978), ”On the Pooling of Time Series and Cross-section Data, " Econometrica, 46, 69-85.

Myers, S. C. and S. Turnbull, (1977), "Determinants of Corporate Bor rowing,” Journal of Financial Economics, Vol. 5, 147-175.

Qian, G. and Wang, D. (1999), "Factors that affect performance of U.S. small and medium sized technology-based enterprises: Does multinationality matters?", Journal of Business and Entrepreneurship, 11(2), pp. 119.

Qian, Gongming (2002) " Multinationality, product diversification, and profitability of emerging US small- and medium-sized enterprises ", Journal of Business Venturing, 17 (6),611-33.

Ravichandran, T., Liu, Y., Han, S. and Hasan, I. (2009) "Diversification and firm performance: Exploring the moderating effects of information technology spending", Journal of Management Information Systems, 25(4),205-240.

Rouse, W. B. and K. R. Boff (1998), "R\&D/technology management: A framework for putting technology to work," IEEE Transactions on Systems, Man and Cybernetics, 28(4), 290297.

Rugman, A. M. and Verbeke, A. (2002), "Edith penrose's contribution to the resource-based view of strategic management", Strategic Management Journal, 23(8), 769-780.

Scherer, F., M., and D., Ross, (1965), Industrial Market Structure and Economic Performance, Bosto, Hougton Mifflin, 279-299.

Shah, S. Z. A., Andrew W. Stark , and Saeed Akbar, (2009), The value relevance of major media advertising expenditures: Some U.K. evidence, The International Journal of Accounting, please provide volume and issue 20.

Shih-Yung Wei, Tina C. Chiao, Xiu-Wen Ye, (2017), “The Clustering Analysis of Corporate Ownership and Control Contestability based on Shapley Value", Cluster Computing, 20(3), 2703-2723.

Shivdasani and Yermack, (1999), "CEO involvement in the selection of new board members: An

Copyright (C) 2020, Journal of Advanced Research in Economics and Administrative Sciences (JAREAS), Under a 
empirical analysis," Journal of Finance, 54, 1829-1854.

Sriram, V. and H. J. Sapienza (1991), "An empirical investigation of the role of marketing for small exporters," Journal of Small Business Management, 29(4),.33-43.

Stulz R, (1990)," Managetrial Control of Voting Rights: Financial Poli cies and Market for Corporate Control," Journal of Financial Economics, 20, 25-54.

Titman, S. and R. Wessels, (1988), “The Determinants of Capital Struc ture Choice,"Journal of Finance. , 43 (1), 1-19.

Wernerfelt, B. (1984), "A resource-based view of the firm", Strategic Management Journal, 5(2), 171-180.

Yeh, Y. H., T. S. Lee, and T. Woidtke, (2001),"'Family Control and Corporate Governance: Evidence from Taiwan," International Review of Finance, 2, 21-48.

Yermack, (1996)," Higher market valuation of companies with a small board of directors," Journal of Financial Economics, 40, 185-211.

Yermack, (2004)," Remuneration, Retention, and Reputation Incen tives for Outside Directors," Journal of Finance, Vol. 5, 2281-2308.

Zeng, S. X., Xie, X. M., Tam, C. M. and Wan, T. W. (2009), "Relationships between business factors and performance in internationalization An empirical study in China", Management Decision, 47(2), 308-329. 